\title{
GLOBAL EXISTENCE, EXPONENTIAL DECAY AND FINITE TIME BLOW-UP OF SOLUTIONS FOR A CLASS OF SEMILINEAR PSEUDO-PARABOLIC EQUATIONS WITH CONICAL DEGENERATION
}

\author{
GANG LI, JIANGYONG YU, AND WENJUN LIU
}

\begin{abstract}
In this paper, we study the semilinear pseudo-parabolic equations $u_{t}-\triangle_{\mathbb{B}} u-\triangle_{\mathbb{B}} u_{t}=$ $|u|^{p-1} u$ on a manifold with conical singularity, where $\triangle_{\mathbb{B}}$ is Fuchsian type Laplace operator investigated with totally characteristic degeneracy on the boundary $x_{1}=0$. Firstly, we discuss the invariant sets and the vacuum isolating behavior of solutions with the help of a family of potential wells. Then, we derive a threshold result of existence and nonexistence of global weak solution: for the low initial energy $J\left(u_{0}\right)<d$, the solution is global in time with $I\left(u_{0}\right)>0$ or $\left\|\nabla_{\mathbb{B}} u_{0}\right\|_{L_{2}^{\frac{n}{2}}(\mathbb{B})}=0$ and blows up in finite time with $I\left(u_{0}\right)<0$; for the critical initial energy $J\left(u_{0}\right)=d$, the solution is global in time with $I\left(u_{0}\right) \geq 0$ and blows up in finite time with $I\left(u_{0}\right)<0$. The decay estimate of the energy functional for the global solution and the estimates of the lifespan of local solution are also given.
\end{abstract}

\section{INTRODUCTION}

In this paper, we consider the following initial-boundary value problem for a class of semilinear pseudoparabolic equation with conical degeneration

$$
\begin{cases}u_{t}-\triangle_{\mathbb{B}} u_{t}-\triangle_{\mathbb{B}} u=|u|^{p-1} u, & x \in \operatorname{int} \mathbb{B}, t>0, \\ u(0)=u_{0}, & x \in \operatorname{int} \mathbb{B}, \\ u=0, & x \in \partial \mathbb{B}, t \geq 0,\end{cases}
$$

where $2<p+1<\frac{2 n}{n-2}=2^{*}$, and $2^{*}$ is the critical cone Sobolev exponents. Here $\mathbb{B}=[0,1) \times X, X$ is an $(n-1)$-dimensional closed compact manifold, which is regarded as the local model near the conical points, and $\partial \mathbb{B}=\{0\} \times X$. Moreover, the operator $\triangle_{\mathbb{B}}$ in (1.1) is defined by $\left(x_{1} \partial_{x_{1}}\right)^{2}+\partial_{x_{2}}^{2}+\cdots+\partial_{x_{n}}^{2}$, which is an elliptic operator with conical degeneration on the boundary $x_{1}=0$ (we also called it Fuchsian type Laplace operator), and corresponding gradient operator is denoted by $\nabla_{\mathbb{B}}=\left(x_{1} \partial_{x_{1}}, \partial_{x_{2}}, \ldots, \partial_{x_{n}}\right)$. Near $\partial \mathbb{B}$ we will use coordinates $\left(x_{1}, x^{\prime}\right)=\left(x_{1}, x_{2}, \ldots, x_{n}\right)$ for $0 \leq x_{1}<1, x^{\prime} \in X$.

The equation in (1.1) is a important physical model, appears in many applications to natural sciences, such as the unidirectional propagation of nonlinear, dispersive, long waves [2], the aggregation of population [19] and the nonstationary processes in crystalline semiconductors [11].

In the classical case, we have

$$
\begin{cases}u_{t}-\Delta u_{t}-\Delta u=|u|^{p-1} u, & x \in \Omega, t>0, \\ u(0)=u_{0}, & x \in \Omega, \\ u=0, & x \in \partial \Omega, t \geq 0,\end{cases}
$$

where $\Omega$ is an open bounded domain of $\mathbb{R}^{n}$ with smooth boundary $\partial \Omega$ and $\triangle$ is the standard Laplace operator. It's well known that problem (1.2) has been studied by many authors. A powerful technique for

2010 Mathematics Subject Classification. 26D15, 41A55.

Key words and phrases. blow-up; semilinear pseudo-parabolic equations; critical initial energy; conical degeneration. 
treating problem (1.2) is the so called "potential well method", which was established by Sattinger [21], Payne and Sattinger [20], and then improved by Liu and Zhao [16] by introducing a family of potential wells. Recently, there are some interesting results about the global existence and blow-up of solutions for problem (1.2) in [24], in which $\mathrm{Xu}$ and $\mathrm{Su}$ proved the invariance of some sets, global existence, nonexistence and asymptotic behavior of solutions with initial energy $J\left(u_{0}\right) \leq d$ and obtained finite time blow-up with high initial energy $J\left(u_{0}\right)>d$ by comparison principle. In [18], the author obtained a lower bound for blow-up time if $p$ and the initial value satisfy some conditions. For other related works, we refer the readers to $[3,4,9,22,23]$ and the references therein.

In the conical degeneration case, Chen et al. established the corresponding Sobolev inequality and Poincaré inequality on the cone Sobolev spaces in [6]. Then in [5], Chen and Liu proved the existence theorem of global solutions with exponential decay and show the blow-up in finite time of solutions to the parabolic problem

$$
\begin{cases}u_{t}-\triangle_{\mathbb{B}} u=|u|^{p-1} u, & x \in \text { int } \mathbb{B}, t>0 \\ u(0)=u_{0}, & x \in \text { int } \mathbb{B}, \\ u=0, & x \in \partial \mathbb{B}, t \geq 0\end{cases}
$$

where $\mathbb{B}$ is the same as above. In [8], Chen and Liu studied the initial boundary value problem for a class of semilinear edge-degenerate parabolic equations with singular potential term, and derived a threshold of the existence of global solutions with exponential decay, and the blow-up in finite time by introducing a family of potential wells. More works on equations with conical degeneration can be seen in the monograph $[1,10]$ and references therein.

In this paper, we aim to use the improved potential well theory to prove the invariant sets, the vacuum isolating behavior, and the global existence, decay and finite time blow-up of solutions for problem (1.1) in weighted Sobolev space. For our purpose, we introduce a family of potential wells and its corresponding sets, and construct the relation between the existence of solution and the initial data $u_{0}$ via the method of the potential wells. Then, by the usage of Faedo-Galerkin method, the concavity argument and properties of a family of potential wells, we derive a threshold result of existence and nonexistence of global weak solution: for the low initial energy case (i.e., $J\left(u_{0}\right)<d$ ), the solution is global in time with $I\left(u_{0}\right)>0$ or $\left\|\nabla_{\mathbb{B}} u_{0}\right\|_{L_{2}^{\frac{n}{2}}(\mathbb{B})}=0$ and blows up in finite time with $I\left(u_{0}\right)<0$; for the critical initial energy case (i.e., $\left.J\left(u_{0}\right)=d\right)$, the solution is global in time with $I\left(u_{0}\right) \geq 0$ and blows up in finite time with $I\left(u_{0}\right)<0$. The decay estimate of the energy functional for the global solution and the estimates of the lifespan of local solution and lower bound on blow-up time are given by making use of a differential inequality technique.

The outline of this paper are as follows. In Section 2, we recall the cone Sobolev spaces and the corresponding properties. In Section 3, we give some preliminaries about the family of potential wells, after which we discuss the invariant sets and the vacuum isolating behavior of solutions for problem (1.1). In Section 4, we show the global existence, decay and finite time blow-up for problem (1.1) with low initial energy $J\left(u_{0}\right)<d$. In Section 5 , we obtain the global existence, decay and finite time blow-up for problem (1.1) with critical initial energy $J\left(u_{0}\right)=d$. 


\section{Cone Sobolev spaces}

In this section, we recall the manifold with conical singularities and the corresponding cone Sobolev spaces which are introduced in $[6,7]$.

Let $X$ be a closed, compact, $C^{\infty}$ manifold. We set $X^{\triangle}=\overline{\mathbb{R}}_{+} \times X /(\{0\} \times X)$ as a local model interpreted as a cone with the base $X$. Next, we denote $X^{\wedge}=\mathbb{R}_{+} \times X$ as the corresponding open stretched cone with the base $X$.

An $n$-dimensional manifold $B$ with conical singularities is a topological space with a finite subset $B_{0}=\left\{b_{1}, \cdots, b_{M}\right\} \subset B$ of conical singularities, with the following two properties.

(1) $B \backslash B_{0}$ is a $C^{\infty}$ manifold.

(2) Every $b \in B_{0}$ has an open neighbourhood $U$ in $B$, such that there is a homeomorphism $\varphi$ : $U \rightarrow X^{\triangle}$ for some closed compact $C^{\infty}$ manifold $X=X(b)$, and $\varphi$ restricts to a diffeomorphism $\varphi^{\prime}: U \backslash\{b\} \rightarrow X^{\wedge}$.

For simplicity, we assume that the manifold $B$ has only one conical point on the boundary. Thus, near the conical point, we have a stretched manifold $\mathbb{B}$, associated with $B$. Here $\mathbb{B}=[0,1) \times X, \partial \mathbb{B}=\{0\} \times X$ and $X$ is a closed compact manifold of dimension $n-1$. Also, near the conical point, we use the coordinates $\left(x_{1}, x^{\prime}\right)=\left(x_{1}, x_{2}, \ldots, x_{n}\right)$ for $0 \leq x_{1}<1, x^{\prime} \in X$.

Definition 1. Let $\mathbb{B}=[0,1) \times X$ be the stretched manifold of the manifold $B$ with conical singularity. Then the cone Sobolev space $\mathcal{H}_{p}^{m, \gamma}(B)$, for $m \in \mathbb{N}, \gamma \in \mathbb{R}$ and $1<p<+\infty$, is defined as

$$
\mathcal{H}_{p}^{m, \gamma}(\mathbb{B})=\left\{u \in W_{l o c}^{m, p}(\operatorname{int} \mathbb{B}) \mid \omega u \in \mathcal{H}_{p}^{m, \gamma}\left(X^{\wedge}\right)\right\}
$$

for any cut-off function $\omega$, supported by a collar neighborhood of $(0,1) \times \partial \mathbb{B}$. Moreover, the subspace $\mathcal{H}_{p, 0}^{m, \gamma}(\mathbb{B})$ of $\mathcal{H}_{p}^{m, \gamma}(\mathbb{B})$ is defined by

$$
\mathcal{H}_{p, 0}^{m, \gamma}(\mathbb{B}):=[\omega] \mathcal{H}_{p, 0}^{m, \gamma}\left(X^{\wedge}\right)+[1-\omega] W_{0}^{m, p}(\operatorname{int} \mathbb{B}),
$$

where $W_{0}^{m, p}($ int $\mathbb{B})$ denotes the closure of $C_{0}^{\infty}($ int $\mathbb{B})$ in Sobolev spaces $W^{m, p}(\bar{X})$ when $\bar{X}$ is a closed compact $C^{\infty}$ manifold of dimension $n$ that containing $\mathbb{B}$ as a submanifold with boundary.

Definition 2. Let $\mathbb{B}=[0,1) \times X$. We say $u(x) \in L_{p}^{\gamma}(\mathbb{B})$ with $1<p<+\infty$ and $\gamma \in \mathbb{R}$ if

$$
\|u\|_{L_{p}^{\gamma}(\mathbb{B})}^{p}=\int_{\mathbb{B}} x_{1}^{n}\left|x_{1}^{-\gamma} u(x)\right|^{p} \frac{d x_{1}}{x_{1}} d x^{\prime}<+\infty .
$$

Observe that if $u(x) \in L_{p}^{\frac{n}{p}}(\mathbb{B}), v(x) \in L_{q}^{\frac{n}{q}}(\mathbb{B})$ with $p, q \in(1, \infty)$ and $\frac{1}{p}+\frac{1}{q}=1$, then we have the following Hölder's inequality

$$
\int_{\mathbb{B}}|u(x) v(x)| \frac{d x_{1}}{x_{1}} d x^{\prime} \leq\left(\int_{\mathbb{B}}|u(x)|^{p} \frac{d x_{1}}{x_{1}} d x^{\prime}\right)^{\frac{1}{p}}\left(\int_{\mathbb{B}}|v(x)|^{q} \frac{d x_{1}}{x_{1}} d x^{\prime}\right)^{\frac{1}{q}} .
$$

In the sequel, for convenience we denote

$$
(u, v)_{2}=\int_{\mathbb{B}} u(x) v(x) \frac{d x_{1}}{x_{1}} d x^{\prime}, \quad\|u\|_{L_{p}^{\frac{n}{p}}(\mathbb{B})}^{p}=\int_{\mathbb{B}}|u(x)|^{p} \frac{d x_{1}}{x_{1}} d x^{\prime} .
$$


Proposition 1 ([6], Poincaré inequality). Let $\mathbb{B}=[0,1) \times X$ be a bounded subspace in $\mathbb{R}_{+}^{n}$ with $X \subset \mathbb{R}^{n-1}$, and $1<p<+\infty, \gamma \in \mathbb{R}$. If $u(x) \in \mathcal{H}_{p, 0}^{1, \gamma}(\mathbb{B})$, then

$$
\|u(x)\|_{L_{p}^{\gamma}(\mathbb{B})} \leq c\left\|\nabla_{\mathbb{B}} u(x)\right\|_{L_{p}^{\gamma}(\mathbb{B})},
$$

where $\nabla_{\mathbb{B}}=\left(x_{1} \partial_{x_{1}}, \partial_{x_{2}}, \ldots, \partial_{x_{n}}\right)$, and the constant $c$ depends only on $\mathbb{B}$.

Proposition $2\left([7]\right.$, Proposition 3.3). For $1<p<2^{*}$, the embedding $\mathcal{H}_{2,0}^{1, \frac{n}{2}}(\mathbb{B}) \hookrightarrow \mathcal{H}_{p, 0}^{0, \frac{n}{p}}(\mathbb{B})$ is continuous.

Similar to the classical case, we introduced the following functionals on cone Sobolev space $\mathcal{H}_{2,0}^{1, \frac{n}{2}}(\mathbb{B})$ :

$$
\begin{gathered}
J(u)=\frac{1}{2} \int_{\mathbb{B}}\left|\nabla_{\mathbb{B}} u\right|^{2} \frac{d x_{1}}{x_{1}} d x^{\prime}-\frac{1}{p+1} \int_{\mathbb{B}}|u|^{p+1} \frac{d x_{1}}{x_{1}} d x^{\prime}, \\
I(u)=\int_{\mathbb{B}}\left|\nabla_{\mathbb{B}} u\right|^{2} \frac{d x_{1}}{x_{1}} d x^{\prime}-\int_{\mathbb{B}}|u|^{p+1} \frac{d x_{1}}{x_{1}} d x^{\prime} .
\end{gathered}
$$

Then $J(u)$ and $I(u)$ are well-defined and belong to $\mathcal{C}^{\prime}\left(\mathcal{H}_{2,0}^{1, \frac{n}{2}}(\mathbb{B})\right)$. We introduce the potential well

$$
W=\left\{u \in \mathcal{H}_{2,0}^{1, \frac{n}{2}}(\mathbb{B}) \mid I(u)>0, J(u)<d\right\} \cup\{0\}
$$

and the outside sets of the corresponding potential well

$$
V=\left\{u \in \mathcal{H}_{2,0}^{1, \frac{n}{2}}(\mathbb{B}) \mid I(u)<0, J(u)<d\right\} .
$$

We define the potential well depth $d$ as

$$
d=\inf \left\{\sup _{\lambda \geq 0} J(\lambda u), u \in \mathcal{H}_{2,0}^{1, \frac{n}{2}}(\mathbb{B}), \int_{\mathbb{B}}\left|\nabla_{\mathbb{B}} u\right|^{2} \frac{d x_{1}}{x_{1}} d x^{\prime} \neq 0\right\},
$$

and the Nehari manifold

$$
\mathcal{N}=\left\{\left.u \in \mathcal{H}_{2,0}^{1, \frac{n}{2}}(\mathbb{B})\left|I(u)=0, \int_{\mathbb{B}}\right| \nabla_{\mathbb{B}} u\right|^{2} \frac{d x_{1}}{x_{1}} d x^{\prime} \neq 0\right\} .
$$

Similar to the results in [24], one has $0<d=\inf _{u \in \mathcal{N}} J(u)$.

\section{INVARIANT SETS AND VACUUM ISOLATING}

In this section, we shall introduce a family of Nehari functionals $I_{\delta}(u)$ in cone Sobolev spaces, the family of potential wells sets and give the corresponding lemmas, which will help us to demonstrate the invariant sets and the vacuum isolating behavior of solutions for problem (1.1).

3.1. Properties of potential wells. In this subsection, we shall introduce a family of potential wells $W_{\delta}$, its corresponding sets $V_{\delta}$ and give a series of their properties which are useful in the proof of our main results.

Lemma 3.1. Let $u \in \mathcal{H}_{2,0}^{1, \frac{n}{2}}(\mathbb{B})$, and $\left\|\nabla_{\mathbb{B}} u\right\|_{2}^{2} \neq 0$. Then:

(1) $\lim _{\lambda \rightarrow 0} J(\lambda u)=0, \lim _{\lambda \rightarrow+\infty} J(\lambda u)=-\infty$.

(2) On the interval $0<\lambda<\infty$, there exists a unique $\lambda^{*}=\lambda^{*}(u)$, such that $\left.\frac{d}{d \lambda} J(\lambda u)\right|_{\lambda=\lambda^{*}}=0$.

(3) $J(\lambda u)$ is increasing on $0 \leq \lambda \leq \lambda^{*}$, decreasing on $\lambda^{*} \leq \lambda<\infty$ and takes the maximum at $\lambda=\lambda^{*}$.

(4) $I(\lambda u)>0$ for $0<\lambda<\lambda^{*}, I(\lambda u)<0$ for $\lambda^{*}<\lambda<\infty$, and $I\left(\lambda^{*} u\right)=0$. 
Proof. (1) From the definition of $J(u)$, we know

$$
J(\lambda u)=\frac{\lambda^{2}}{2} \int_{\mathbb{B}}\left|\nabla_{\mathbb{B}} u\right|^{2} \frac{d x_{1}}{x_{1}} d x^{\prime}-\frac{\lambda^{p+1}}{p+1} \int_{\mathbb{B}}|u|^{p+1} \frac{d x_{1}}{x_{1}} d x^{\prime},
$$

which gives

$$
\lim _{\lambda \rightarrow 0} J(\lambda u)=0
$$

and

$$
\lim _{\lambda \rightarrow+\infty} J(\lambda u)=-\infty
$$

(2) An easy calculation shows that

$$
\frac{d}{d \lambda} J(\lambda u)=\lambda\left(\int_{\mathbb{B}}\left|\nabla_{\mathbb{B}} u\right|^{2} \frac{d x_{1}}{x_{1}} d x^{\prime}\right)-\lambda^{p} \int_{\mathbb{B}}|u|^{p+1} \frac{d x_{1}}{x_{1}} d x^{\prime} .
$$

Let $\lambda^{*}=\left(\frac{\left\|\nabla_{\mathbb{B}} u\right\|_{L_{2}^{\frac{n}{2}} \mathbb{B}}^{2}}{\int_{\mathbb{B}}|u|^{p+1} \frac{d x_{1}}{x_{1}} d x^{\prime}}\right)^{\frac{1}{p-1}}$, then $\left.\frac{d J(\lambda u)}{d \lambda}\right|_{\lambda=\lambda^{*}}=0$.

(3) From

$$
\frac{\partial^{2} J(\lambda u)}{\partial \lambda^{2}}=\left\|\nabla_{\mathbb{B}} u\right\|_{L_{2}^{\frac{n}{2}}}^{2}-p \lambda^{p-1} \int_{\mathbb{B}}|u|^{p+1} \frac{d x_{1}}{x_{1}} d x^{\prime}
$$

we have

$$
\left.\frac{\partial^{2} J(\lambda u)}{\partial \lambda^{2}}\right|_{\lambda=\lambda^{*}}=\left\|\nabla_{\mathbb{B}} u\right\|_{L_{2}^{\frac{n}{2}(\mathbb{B})}}^{2}-p\left\|\nabla_{\mathbb{B}} u\right\|_{L_{2}^{\frac{n}{2}}(\mathbb{B})}^{2}<0, \quad \text { as } p>1 .
$$

So, the conclusion of (3) holds.

(4) The conclusion follows from

$$
I(\lambda u)=\lambda^{2} \int_{\mathbb{B}}\left|\nabla_{\mathbb{B}} u\right|^{2} \frac{d x_{1}}{x_{1}} d x^{\prime}-\lambda^{p+1} \int_{\mathbb{B}}|u|^{p+1} \frac{d x_{1}}{x_{1}} d x^{\prime}=\lambda \frac{d}{d \lambda} J(\lambda u) .
$$

Hence, when $0<\lambda<\lambda^{*}, I(\lambda u)>0$; when $\lambda^{*}<\lambda<\infty, I(\lambda u)<0$; when $\lambda=\lambda^{*}, I(\lambda u)=0$.

Assume that $u \in \mathcal{H}_{2,0}^{1, \frac{n}{2}}(\mathbb{B}),\left\|\nabla_{\mathbb{B}} u\right\|_{L_{2}^{\frac{n}{2}}(\mathbb{B})} \neq 0$, we denote $C_{*}=\sup \left\{\frac{\|u\|_{\frac{n}{L_{p+1}}(\mathbb{B})}}{\left\|\nabla_{\mathbb{B}} u\right\|_{L_{2}^{\frac{n}{2}}(\mathbb{B})}}\right\}$, which can be obtained from Proposition 1 and Proposition 2.

For $\delta>0$, we define in cone Sobolev spaces a set of Nehari functionals

$$
I_{\delta}(u)=\delta \int_{\mathbb{B}}\left|\nabla_{\mathbb{B}} u\right|^{2} \frac{d x_{1}}{x_{1}} d x^{\prime}-\int_{\mathbb{B}}|u|^{p+1} \frac{d x_{1}}{x_{1}} d x^{\prime},
$$

and denote

$$
\gamma(\delta)=\left(\frac{\delta}{C_{*}^{p+1}}\right)^{\frac{1}{p-1}}
$$

Lemma 3.2. Let $u_{0} \in \mathcal{H}_{2,0}^{1, \frac{n}{2}}(\mathbb{B})$ :

(1) If $0<\left\|\nabla_{\mathbb{B}} u\right\|_{L_{2}^{\frac{n}{2}}(\mathbb{B})}<\gamma(\delta)$, then $I_{\delta}(u)>0$. In particular, if $0<\left\|\nabla_{\mathbb{B}} u\right\|_{L_{2}^{\frac{n}{2}}(\mathbb{B})}<\gamma(1)$, then $I(u)>0$.

(2) If $I_{\delta}(u)<0$, then $\left\|\nabla_{\mathbb{B}} u\right\|_{L_{2}^{\frac{n}{2}}(\mathbb{B})}>\gamma(\delta)$. In particular, if $I(u)<0$, then $\left\|\nabla_{\mathbb{B}} u\right\|_{L_{2}^{\frac{n}{2}}(\mathbb{B})}>\gamma(1)$.

(3) If $I_{\delta}(u)=0$, then $\left\|\nabla_{\mathbb{B}} u\right\|_{L_{2}^{\frac{n}{2}}(\mathbb{B})} \geq \gamma(\delta)$ or $\left\|\nabla_{\mathbb{B}} u\right\|_{L_{2}^{\frac{n}{2}}(\mathbb{B})}=0$. 
(4) If $I_{\delta}(u)=0$ and $\left\|\nabla_{\mathbb{B}} u\right\|_{L_{2}^{\frac{n}{2}(\mathbb{B})}} \neq 0$, then $J(u)>0$ for $0<\delta<\frac{p+1}{2}, J(u)=0$ for $\delta=\frac{p+1}{2}$, $J(u)>0$ for $\delta>\frac{p+1}{2}$.

Proof. (1) From $0<\|\nabla u\|_{L_{2}^{\frac{n}{2}}(\mathbb{B})}<\gamma(\delta)$, we have

then

$$
\begin{aligned}
\int_{\mathbb{B}}|u|^{p+1} \frac{d x_{1}}{x_{1}} d x^{\prime} & =\int_{\mathbb{B}} x_{1}^{n}\left|x_{1}^{-\frac{n}{p+1}} u\right|^{p+1} \frac{d x_{1}}{x_{1}} d x^{\prime}=\|u\|_{L_{p+1}^{p+1}(\mathbb{B})}^{p+1} \\
& \leq C_{*}^{p+1}\left\|\nabla_{\mathbb{B}} u\right\|_{L_{2}^{\frac{n}{2}}(\mathbb{B})}^{p+1}=C_{*}^{p+1}\left\|\nabla_{\mathbb{B}} u\right\|_{L_{2}^{\frac{n}{2}}(\mathbb{B})}^{p-1}\left\|\nabla_{\mathbb{B}} u\right\|_{L_{2}^{\frac{n}{2}}(\mathbb{B})}^{2} \\
& <\delta\left\|\nabla_{\mathbb{B}} u\right\|_{L_{2}^{\frac{n}{2}}(\mathbb{B})}^{2},
\end{aligned}
$$

$$
I_{\delta}=\delta\left\|\nabla_{\mathbb{B}} u\right\|_{L_{2}^{\frac{n}{2}(\mathbb{B})}}^{2}-\int_{\mathbb{B}}|u|^{p+1} \frac{d x_{1}}{x_{1}} d x^{\prime}>0 .
$$

(2) Notice that $I_{\delta}(u)<0$, then we have $\left\|\nabla_{\mathbb{B}} u\right\|_{L_{2}^{\frac{n}{2}(\mathbb{B})}}^{2} \neq 0$. And we have

then

$$
\delta\left\|\nabla_{\mathbb{B}} u\right\|_{L_{2}^{\frac{n}{2}}(\mathbb{B})}<\int_{\mathbb{B}}|u|^{p+1} \frac{d x_{1}}{x_{1}} d x^{\prime} \leq C_{*}^{p+1}\left\|\nabla_{\mathbb{B}} u\right\|_{L_{2}^{\frac{n}{2}(\mathbb{B})}}^{p+1}=C_{*}^{p+1}\left\|\nabla_{\mathbb{B}} u\right\|_{L_{2}^{\frac{n}{2}}(\mathbb{B})}^{p-1}\left\|\nabla_{\mathbb{B}} u\right\|_{L_{2}^{\frac{n}{2}}(\mathbb{B})}^{2},
$$

$$
\left\|\nabla_{\mathbb{B}} u\right\|_{L_{2}^{\frac{n}{2}}(\mathbb{B})}>\gamma(\delta) .
$$

(3) From $I_{\delta}(u)=0$, we have

$$
\delta\left\|\nabla_{\mathbb{B}} u\right\|_{L_{2}^{\frac{n}{2}(\mathbb{B})}}^{2}=\int_{\mathbb{B}}|u|^{p+1} \frac{d x_{1}}{x_{1}} d x^{\prime}
$$

If $\left\|\nabla_{\mathbb{B}} u\right\|_{L_{2}^{\frac{n}{2}(\mathbb{B})}}=0$, then from

we have

$$
\int_{\mathbb{B}}|u|^{p+1} \frac{d x_{1}}{x_{1}} d x^{\prime} \leq C_{*}^{p+1}\left\|\nabla_{\mathbb{B}} u\right\|_{L_{2}^{\frac{n}{2}}}^{p+1}
$$

$$
\int_{\mathbb{B}}|u|^{p+1} \frac{d x_{1}}{x_{1}} d x^{\prime}=0
$$

So when $\left\|\nabla_{\mathbb{B}} u\right\|_{L_{2}^{\frac{n}{2}}(\mathbb{B})}=0$, satisfies. If $\left\|\nabla_{\mathbb{B}} u\right\|_{L_{2}^{\frac{n}{2}(\mathbb{B})}} \neq 0$ and $I_{\delta}(u)=0$, then

So, we have

$$
\delta\left\|\nabla_{\mathbb{B}} u\right\|_{L_{2}^{\frac{n}{2}(\mathbb{B})}}^{2}=\int_{\mathbb{B}}|u|^{p+1} \frac{d x_{1}}{x_{1}} d x^{\prime} \leq C_{*}^{p+1}\left\|\nabla_{\mathbb{B}} u\right\|_{L_{2}^{\frac{n}{2}}}^{p+1}=C_{*}^{p+1}\left\|\nabla_{\mathbb{B}} u\right\|_{L_{2}^{\frac{n}{2}}(\mathbb{B})}^{p-1}\left\|\nabla_{\mathbb{B}} u\right\|_{L_{2}^{\frac{n}{2}}(\mathbb{B})}^{2} .
$$

$$
\left\|\nabla_{\mathbb{B}} u\right\|_{L_{2}^{\frac{n}{2}(\mathbb{B})}} \geq\left(\frac{\delta}{C_{*}^{p+1}}\right)^{\frac{1}{p-1}}=\gamma(\delta) .
$$

(4) By $I_{\delta}(u)=0$ and $\left\|\nabla_{\mathbb{B}} u\right\|_{L_{2}^{\frac{n}{2}(\mathbb{B})}}^{2}>0$, we have

$$
\begin{aligned}
J(u) & =\frac{1}{2} \int_{\mathbb{B}}\left|\nabla_{\mathbb{B}} u\right|^{2} \frac{d x_{1}}{x_{1}} d x^{\prime}-\frac{1}{p+1} \int_{\mathbb{B}}|u|^{p+1} \frac{d x_{1}}{x_{1}} d x^{\prime} \\
& =\left(\frac{1}{2}-\frac{\delta}{p+1}\right)\left\|\nabla_{\mathbb{B}} u\right\|_{L_{2}^{\frac{n}{2}(\mathbb{B})}}^{2}+\frac{1}{p+1} I_{\delta} \\
& =\left(\frac{1}{2}-\frac{\delta}{p+1}\right)\left\|\nabla_{\mathbb{B}} u\right\|_{L_{2}^{\frac{n}{2}}(\mathbb{B})}^{2} .
\end{aligned}
$$

So, the conclusion of (4) holds. 
Now, for $\delta>0$, we define the depth of a family of potential wells as follows

$$
d(\delta)=\inf _{u \in \mathcal{N}_{\delta}} J(u)
$$

where

$$
\mathcal{N}_{\delta}=\left\{\left.u \in \mathcal{H}_{2,0}^{1, \frac{n}{2}}(\mathbb{B})\left|I_{\delta}(u)=0, \int_{\mathbb{B}}\right| \nabla_{\mathbb{B}} u\right|^{2} \frac{d x_{1}}{x_{1}} d x^{\prime} \neq 0\right\} .
$$

Then, the depth $d(\delta)$ and its expression can be estimated. Additionally, we show that how $d(\delta)$ behaves with respect to $\delta$ in the following lemma.

Lemma 3.3. $d(\delta)$ satisfies the following properties:

(1) $d(\delta) \geq a(\delta) \gamma^{2}(\delta)$ for $a(\delta)=\frac{1}{2}-\frac{\delta}{p+1}$ and $0<\delta<\frac{p+1}{2}$. Moreover, we have

$$
d(\delta)=\inf _{u \in \mathcal{N}_{\delta}} J(u)=\delta^{\frac{2}{p-1}}\left(\frac{1}{2}-\frac{\delta}{p+1}\right) \frac{2(p+1)}{p-1} d, \quad 0<\delta<\frac{p+1}{2} .
$$

(2) $\lim _{\delta \rightarrow 0} d(\delta)=0, d\left(\frac{p+1}{2}\right)=0$, and $d(\delta)<0$ for $\delta>\frac{p+1}{2}$.

(3) $d(\delta)$ is increasing on $0<\delta \leq 1$, decreasing on $1 \leq \delta \leq \frac{p+1}{2}$ and takes the maximum $d=d(1)$ at $\delta=1$.

Proof. (1) Let $u \in \mathcal{N}_{\delta}$, then $I_{\delta}(u)=0$. By Lemma $3.2(3)$, we have $\left\|\nabla_{\mathbb{B}} u\right\|_{L_{2}^{\frac{n}{2}(\mathbb{B})}} \geq \gamma(\delta)$. Hence from

$$
\begin{aligned}
J(u) & =\left(\frac{1}{2}-\frac{\delta}{p+1}\right)\left\|\nabla_{\mathbb{B}} u\right\|_{L_{2}^{\frac{n}{2}}(\mathbb{B})}^{2}+\frac{1}{p+1} I_{\delta}(u) \\
& =a(\delta)\left\|\nabla_{\mathbb{B}} u\right\|_{L_{2}^{\frac{n}{2}}(\mathbb{B})}^{2} \\
& \geq a(\delta) \gamma^{2}(\delta),
\end{aligned}
$$

we have

$$
d(\delta)=\inf _{u \in \delta} J(u) \geq a(\delta) \gamma^{2}(\delta) .
$$

If $\delta>0, \tilde{u} \in \mathcal{N}_{\delta}$ is a minimizer of $d(\delta)=\inf _{u \in \mathcal{N}_{\delta}} J(u)$, i.e., $J(\tilde{u})=d(\delta)$. In this case, we define $\lambda=\lambda(\delta)$ by $\left\|\nabla_{\mathbb{B}}(\lambda \tilde{u})\right\|_{L_{2}^{\frac{n}{2}}(\mathbb{B})}^{2}=\int_{\mathbb{B}}|\lambda \tilde{u}|^{p+1} \frac{d x_{1}}{x_{1}} d x^{\prime}$. Then for each $\delta>0$,

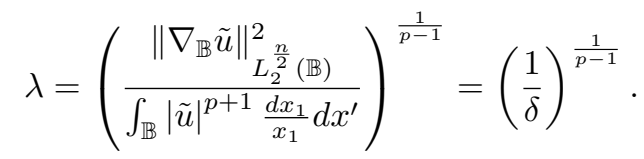

Thus for $\lambda \tilde{u} \in \mathcal{N}_{\delta}$, we get from the definition of $d$ that

$$
\begin{aligned}
d \leq J(\lambda \tilde{u}) & =\frac{\lambda^{2}}{2}\left\|\nabla_{\mathbb{B}} \tilde{u}\right\|_{L_{2}^{\frac{n}{2}}(\mathbb{B})}^{2}-\frac{\lambda^{p+1}}{p+1} \int_{\mathbb{B}}|\tilde{u}|^{p+1} \frac{d x_{1}}{x_{1}} d x^{\prime} \\
& =\frac{1}{2}\left(\frac{1}{\delta}\right)^{\frac{2}{p-1}}\left\|\nabla_{\mathbb{B}} \tilde{u}\right\|_{L_{2}^{\frac{n}{2}}(\mathbb{B})}^{2}-\frac{1}{p+1}\left(\frac{1}{\delta}\right)^{\frac{p+1}{p-1}} \int_{\mathbb{B}}|\tilde{u}|^{p+1} \frac{d x_{1}}{x_{1}} d x^{\prime} \\
& =\left(\frac{1}{\delta}\right)^{\frac{2}{p-1}}\left(\frac{1}{2}\left\|\nabla_{\mathbb{B}} \tilde{u}\right\|_{L_{2}^{\frac{n}{2}}(\mathbb{B})}^{2}-\frac{1}{(p+1) \delta} \int_{\mathbb{B}}|\tilde{u}|^{p+1} \frac{d x_{1}}{x_{1}} d x^{\prime}\right) \\
& =\left(\frac{1}{\delta}\right)^{\frac{2}{p-1}} \frac{p-1}{2(p+1)}\left\|\nabla_{\mathbb{B}} \tilde{u}\right\|_{L_{2}^{\frac{n}{2}}(\mathbb{B})}^{2}
\end{aligned}
$$


Note that $d(\delta)=J(\tilde{u})=\left(\frac{1}{2}-\frac{\delta}{p+1}\right)\left\|\nabla_{\mathbb{B}} \tilde{u}\right\|_{L_{2}^{\frac{n}{2}(\mathbb{B})}}^{2}$, we get

which implies

$$
d \leq\left(\frac{1}{\delta}\right)^{\frac{2}{p-1}} \frac{p-1}{2(p+1)}\left(\frac{1}{2}-\frac{\delta}{p+1}\right)^{-1} d(\delta)
$$

$$
d(\delta) \geq \delta^{\frac{2}{p-1}}\left(\frac{1}{2}-\frac{\delta}{p+1}\right) \frac{2(p+1)}{p-1} d, \quad 0<\delta<\frac{p+1}{2} .
$$

If $\delta>0$, and $\bar{u} \in \mathcal{N}$ is a minimizer of $d=\inf _{u \in \mathcal{N}} J(u)$, i.e., $J(\bar{u})=d$. In this case, we define $\lambda=\lambda(\delta)$ by $\delta\left\|\nabla_{\mathbb{B}}(\lambda \bar{u})\right\|_{L_{2}^{\frac{n}{2}(\mathbb{B})}}^{2}=\int_{\mathbb{B}}|\lambda \bar{u}|^{p+1} \frac{d x_{1}}{x_{1}} d x^{\prime}$. Then for each $\delta>0$,

$$
\lambda=\left(\frac{\delta\left\|\nabla_{\mathbb{B}} \bar{u}\right\|_{L_{2}^{\frac{n}{2}}(\mathbb{B})}^{2}}{\int_{\mathbb{B}}|\bar{u}|^{p+1} \frac{d x_{1}}{x_{1}} d x^{\prime}}\right)^{\frac{1}{p-1}}=\delta^{\frac{1}{p-1}} .
$$

Thus, for $\lambda \bar{u} \in \mathcal{N}_{\delta}$, we get from the definition of $d(\delta)$ that

$$
d(\delta) \leq J(\lambda \bar{u})=\delta^{\frac{2}{p-1}}\left(\frac{1}{2}-\frac{\delta}{p+1}\right)\left\|\nabla_{\mathbb{B}} \bar{u}\right\|_{L_{2}^{\frac{n}{2}}(\mathbb{B})}^{2} .
$$

Note that $d=J(\bar{u})=\left(\frac{1}{2}-\frac{1}{p+1}\right)\left\|\nabla_{\mathbb{B}} \bar{u}\right\|_{L_{2}^{\frac{n}{2}}(\mathbb{B})}^{2}$, we get

$$
d(\delta) \leq \delta^{\frac{2}{p-1}}\left(\frac{1}{2}-\frac{\delta}{p+1}\right) \frac{2(p+1)}{p-1} d, \quad 0<\delta<\frac{p+1}{2} .
$$

By (3.3) and (3.4), we have

$$
d(\delta)=\delta^{\frac{2}{p-1}}\left(\frac{1}{2}-\frac{\delta}{p+1}\right) \frac{2(p+1)}{p-1} d, \quad 0<\delta<\frac{p+1}{2} .
$$

(2) and (3) follow from (3.5).

Lemma 3.4. Let $u_{0} \in \mathcal{H}_{2,0}^{1, \frac{n}{2}}(\mathbb{B})$ and $0<\delta<\frac{p+1}{2}$. Assume that $J(u) \leq d(\delta)$ :

(1) If $I_{\delta}(u)>0$, then $\left\|\nabla_{\mathbb{B}} u\right\|_{L_{2}^{\frac{n}{2}(\mathbb{B})}}^{2}<\frac{d(\delta)}{a(\delta)}$, where $a(\delta)=\frac{1}{2}-\frac{\delta}{p+1}$. In particular, if $J(u) \leq d$ and $I(u)>0$, then $\left\|\nabla_{\mathbb{B}} u\right\|_{L_{2}^{\frac{n}{2}(\mathbb{B})}}^{2}<\frac{2(p+1)}{p-1} d$.

(2) If $\left\|\nabla_{\mathbb{B}} u\right\|_{L_{2}^{\frac{n}{2}(\mathbb{B})}}^{2}>\frac{d(\delta)}{a(\delta)}$, then $I_{\delta}(u)<0$. In particular, if $J(u) \leq d$ and $\left\|\nabla_{\mathbb{B}} u\right\|_{L_{2}^{\frac{n}{2}}(\mathbb{B})}^{2}>\frac{2(p+1)}{p-1} d$, then $I(u)<0$.

(3) If $I_{\delta}(u)=0$, then $\left\|\nabla_{\mathbb{B}} u\right\|_{L_{2}^{\frac{n}{2}(\mathbb{B})}}^{2} \leq \frac{d(\delta)}{a(\delta)}$. In particular, if $J(u) \leq d$ and $I(u)=0$, then $\left\|\nabla_{\mathbb{B}} u\right\|_{L_{2}^{\frac{n}{2}(\mathbb{B})}}^{2} \leq \frac{2(p+1)}{p-1} d$.

Proof. (1) For $0<\delta<\frac{p+1}{2}$, we have

$$
a(\delta)\left\|\nabla_{\mathbb{B}} u\right\|_{L_{2}^{\frac{n}{2}(\mathbb{B})}}^{2}+\frac{1}{p+1} I_{\delta}(u)=J(u) \leq d(\delta) .
$$

Then $a(\delta)\left\|\nabla_{\mathbb{B}} u\right\|_{L_{2}^{\frac{n}{2}(\mathbb{B})}}^{2}<d(\delta)$, i.e., $\left\|\nabla_{\mathbb{B}} u\right\|_{L_{2}^{\frac{n}{2}}(\mathbb{B})}^{2}<\frac{d(\delta)}{a(\delta)}$. If $J(u) \leq d$ and $I(u)>0$, we can get

$$
\left(\frac{1}{2}-\frac{1}{p+1}\right)\left\|\nabla_{\mathbb{B}} u\right\|_{L_{2}^{\frac{n}{2}}(\mathbb{B})}^{2}<\left(\frac{1}{2}-\frac{1}{p+1}\right)\left\|\nabla_{\mathbb{B}} u\right\|_{L_{2}^{\frac{n}{2}}(\mathbb{B})}^{2}+\frac{1}{p+1} I(u) \leq d .
$$


Therefore, we obtain

$$
\left\|\nabla_{\mathbb{B}} u\right\|_{L_{2}^{\frac{n}{2}(\mathbb{B})}}^{2}<\frac{2(p+1)}{p-1} d .
$$

(2) If $\left\|\nabla_{\mathbb{B}} u\right\|_{L_{2}^{\frac{n}{2}}}^{2}>\frac{d(\delta)}{a(\delta)}$, then from $(3.6)$, we get $I_{\delta}(u)<0$. If $J(u) \leq d$, we have $I(u) \leq(p+1) d-$ $\frac{p-1}{2}\left\|\nabla_{\mathbb{B}} u\right\|_{L_{2}^{\frac{n}{2}}(\mathbb{B})}^{2}$. By $\left\|\nabla_{\mathbb{B}} u\right\|_{L_{2}^{\frac{n}{2}}(\mathbb{B})}^{2}>\frac{2(p+1)}{p-1} d$, we get $I(u)<(p+1) d-(p+1) d=0$.

(3) From (3.6), we have $\left\|\nabla_{\mathbb{B}} u\right\|_{L_{2}^{\frac{n}{2}}(\mathbb{B})}^{2} \leq \frac{d(\delta)}{a(\delta)}$. If $J(u) \leq d$ and $I(u)=0$, then from

$$
\left(\frac{1}{2}-\frac{1}{p+1}\right)\left\|\nabla_{\mathbb{B}} u\right\|_{L_{2}^{\frac{n}{2}}(\mathbb{B})}^{2}+\frac{1}{p+1} I(u) \leq d,
$$

we have $\left\|\nabla_{\mathbb{B}} u\right\|_{L_{2}^{\frac{n}{2}}(\mathbb{B})}^{2} \leq \frac{2(p+1)}{p-1} d$.

Lemma 3.5. Assume $0<J(u)<d$ for some $u \in \mathcal{H}_{2,0}^{1, \frac{n}{2}}(\mathbb{B})$, and $\delta_{1}<\delta_{2}$ are two roots of equation $d(\delta)=J(u)$. Then the sign of $I_{\delta}(u)$ doesn't change for $\delta_{1}<\delta<\delta_{2}$.

Proof. $J(u)>0$ implies $\left\|\nabla_{\mathbb{B}} u\right\|_{L_{2}^{\frac{n}{2}}(\mathbb{B})} \neq 0$. If the sign of $I_{\delta}(u)$ is changeable for $\delta_{1}<\delta<\delta_{2}$, then we can choose $\bar{\delta} \in\left(\delta_{1}, \delta_{2}\right)$ and $I_{\bar{\delta}}(u)=0$. Therefore, we can have $J(u) \geq d(\bar{\delta})$. From Lemma $3.3(3)$, we have $J(u)=d\left(\delta_{1}\right)=d\left(\delta_{2}\right)<d(\bar{\delta})$, which is contradict with $J(u) \geq d(\bar{\delta})$.

Now, we are in a position to introduce a series of potential wells. For $0<\delta<\frac{p+1}{2}$, we define

$$
\begin{aligned}
& W_{\delta}=\left\{u \in \mathcal{H}_{2,0}^{1, \frac{n}{2}}(\mathbb{B}) \mid I_{\delta}(u)>0, J(u)<d(\delta)\right\} \cup\{0\}, \\
& V_{\delta}=\left\{u \in \mathcal{H}_{2,0}^{1, \frac{n}{2}}(\mathbb{B}) \mid I_{\delta}(u)<0, J(u)<d(\delta)\right\} .
\end{aligned}
$$

From the definition of $W_{\delta}, V_{\delta}$ and Lemma 3.3, we can obtain the following lemmas:

Lemma 3.6. (1) If $0<\delta^{\prime}<\delta^{\prime \prime} \leq 1$, then $W_{\delta^{\prime}} \subset W_{\delta^{\prime \prime}}$.

(2) If $1 \leq \delta^{\prime \prime}<\delta^{\prime}<\frac{p+1}{2}$, then $V_{\delta^{\prime}} \subset V_{\delta^{\prime \prime}}$.

In addition, we define

$$
\begin{aligned}
& B_{\delta}=\left\{u \in \mathcal{H}_{2,0}^{1, \frac{n}{2}}(\mathbb{B}) \mid\left\|\nabla_{\mathbb{B}} u\right\|_{L_{2}^{\frac{n}{2}}(\mathbb{B})}<\gamma(\delta)\right\}, \\
& \bar{B}_{\delta}=B_{\delta} \cup \partial B_{\delta}=\left\{u \in \mathcal{H}_{2,0}^{1, \frac{n}{2}}(\mathbb{B}) \mid\left\|\nabla_{\mathbb{B}} u\right\|_{L_{2}^{\frac{n}{2}}(\mathbb{B})} \leq \gamma(\delta)\right\}, \\
& B_{\delta}^{c}=\left\{u \in \mathcal{H}_{2,0}^{1, \frac{n}{2}}(\mathbb{B}) \mid\left\|\nabla_{\mathbb{B}} u\right\|_{L_{2}^{\frac{n}{2}}(\mathbb{B})}>\gamma(\delta)\right\} .
\end{aligned}
$$

Lemma 3.7. Let $0<\delta<\frac{p+1}{2}$. Then

$$
B_{\gamma_{1}(\delta)} \subset W_{\delta} \subset B_{\gamma_{2}(\delta)}, V_{\delta} \subset B_{\delta}^{c}
$$

where

$$
\begin{aligned}
& B_{\gamma_{1}(\delta)}=\left\{u \in \mathcal{H}_{2,0}^{1, \frac{n}{2}}(\mathbb{B}) \mid\left\|\nabla_{\mathbb{B}} u\right\|_{L_{2}^{\frac{n}{2}}(\mathbb{B})}^{2}<\min \left\{\gamma^{2}(\delta), \gamma_{0}^{2}(\delta)\right\}\right\}, \\
& B_{\gamma_{2}(\delta)}=\left\{u \in \mathcal{H}_{2,0}^{1, \frac{n}{2}}(\mathbb{B}) \mid\left\|\nabla_{\mathbb{B}} u\right\|_{L_{2}^{\frac{n}{2}}(\mathbb{B})}^{2}<\frac{d(\delta)}{a(\delta)}\right\},
\end{aligned}
$$


where $\gamma_{0}(\delta)$ is the unique real root of equation

$$
\frac{1}{2} \gamma^{2}=d(\delta)
$$

Proof. First $\left\|\nabla_{\mathbb{B}} u\right\|_{L_{2}^{\frac{n}{2}}(\mathbb{B})}<\gamma(\delta)$ gives $\left\|\nabla_{\mathbb{B}} u\right\|_{L_{2}^{\frac{n}{2}}(\mathbb{B})}=0$ or $I_{\delta}(u)>0$. On the other hand,

$$
J(u) \leq \frac{1}{2}\left\|\nabla_{\mathbb{B}} u\right\|_{L_{2}^{\frac{n}{2}}(\mathbb{B})}^{2}
$$

and $\left\|\nabla_{\mathbb{B}} u\right\|_{L_{2}^{\frac{n}{2}}(\mathbb{B})}^{2}<\gamma_{0}^{2}(\delta)$ yield $J(u)<d(\delta)$. Hence we have $B_{\gamma_{1}(\delta)} \subset W_{\delta}$. The remainder of this lemma follows from Lemma 3.2 and Lemma 3.4 .

3.2. Invariant sets and Vacuum isolating. In this subsection, we prove the invariance of some sets under the flow of (1.1) and the vacuum isolating behavior of problem (1.1).

Definition 3 (Maximal existence time). Let $u(t)$ be a weak solution of problem (1.1). We define the maximal existence time $T_{\max }$ of $u(t)$ as follows:

(1) If $u(t)$ exists for $0 \leq t<\infty$, then $T_{\max }=+\infty$.

(2) If there exists a $t_{0} \in(0, \infty)$ such that $u(t)$ exists for $0 \leq t<t_{0}$, but doesn't exist at $t=t_{0}$, then $T_{\max }=t_{0}$.

Definition 4 (Weak solution). Function $u=u(x, t)$ is called a weak solution of problem (1.1) on int $\mathbb{B} \times$ $\left[0, T_{\max }\right)$, with $0<T_{\max } \leq+\infty$ being the maximal existence time, if $u \in L^{\infty}\left(0, T_{\max } ; \mathcal{H}_{2,0}^{1, \frac{n}{2}}(\mathbb{B})\right)$ with $u_{t} \in L^{2}\left(0, T_{\max } ; \mathcal{H}_{2,0}^{1, \frac{n}{2}}(\mathbb{B})\right)$ and satisfies problem (1.1) in the distribution sense, i.e.,

(1) $\forall v \in \mathcal{H}_{2,0}^{1, \frac{n}{2}}(\mathbb{B}), t \in\left(0, T_{\max }\right)$,

$$
\left(u_{t}, v\right)_{2}+\left(\nabla_{\mathbb{B}} u, \nabla_{\mathbb{B}} v\right)_{2}+\left(\nabla_{\mathbb{B}} u_{t}, \nabla_{\mathbb{B}} v\right)_{2}=\left(|u|^{p-1} u, v\right)_{2} .
$$

(2) $u(x, 0)=u_{0}(x)$ in $\mathcal{H}_{2,0}^{1, \frac{n}{2}}(\mathbb{B})$.

(3) For $0 \leq t<T_{\max }$,

$$
\int_{0}^{t}\left\|u_{\tau}\right\|_{L_{2}^{\frac{n}{2}}(\mathbb{B})}^{2} d \tau+\int_{0}^{t}\left\|\nabla_{\mathbb{B}} u_{\tau}\right\|_{L_{2}^{\frac{n}{2}(\mathbb{B})}}^{2} d \tau+J(u(t)) \leq J\left(u_{0}\right) .
$$

Now, we discuss the invariance of some sets corresponding to problem (1.1) inspired by the ideas in $[17]$.

Theorem 3.1. Let $u_{0} \in \mathcal{H}_{2,0}^{1, \frac{n}{2}}(\mathbb{B}), 0<e<d, \delta_{1}<\delta_{2}$ be the two roots of equation $d(\delta)=e$. Then:

(1) All weak solutions $u$ of problem (1.1) with $0<J\left(u_{0}\right) \leq$ e belong to $W_{\delta}$ for $\delta_{1}<\delta<\delta_{2}$, $0 \leq t<T_{\text {max }}$, provided $I\left(u_{0}\right)>0$ or $\left\|\nabla_{\mathbb{B}} u_{0}\right\|_{L_{2}^{\frac{n}{2}}(\mathbb{B})}=0$.

(2) All weak solutions $u$ of problem (1.1) with $0<J\left(u_{0}\right) \leq$ e belong to $V_{\delta}$ for $\delta_{1}<\delta<\delta_{2}$, $0 \leq t<$ $T_{\max }$, provided $I\left(u_{0}\right)<0$,

where $T_{\max }$ is the maximal existence time of $u$.

Proof. (1) Let $u(t)$ be any weak solution of problem (1.1) with $J\left(u_{0}\right) \leq e, I\left(u_{0}\right)>0$ or $\left\|\nabla_{\mathbb{B}} u\right\|_{L_{2}^{\frac{n}{2}}(\mathbb{B})}=0$. $T$ is the existence time of $u(t)$. If $\left\|\nabla_{\mathbb{B}} u_{0}\right\|_{L_{2}^{\frac{n}{2}}(\mathbb{B})}=0$, then $u_{0}(x) \in W_{\delta}$. If $I\left(u_{0}\right)>0$, then from Lemma 3.5 , it follows $I_{\delta}\left(u_{0}\right)>0$ and $J\left(u_{0}\right)<d(\delta)$. Then $u_{0}(x) \in W_{\delta}$ for $\delta_{1}<\delta<\delta_{2}$. Next, we should 
prove $u(t) \in W_{\delta}$ for $\delta_{1}<\delta<\delta_{2}$ and $0<t<T_{\max }$. Arguing by contradiction, by the continuity of $I(u)$ we suppose that there must exist a $\delta_{0} \in\left(\delta_{1}, \delta_{2}\right)$ and $t_{0} \in\left(0, T_{\max }\right)$ such that $u\left(t_{0}\right) \in \partial W_{\delta_{0}}$, and $I_{\delta_{0}}\left(u\left(t_{0}\right)\right)=0,\left\|\nabla_{\mathbb{B}} u\right\|_{L_{2}^{\frac{n}{2}}(\mathbb{B})} \neq 0$ or $J\left(u\left(t_{0}\right)\right)=d\left(\delta_{0}\right)$. From

$$
\int_{0}^{t}\left\|u_{\tau}\right\|_{L_{2}^{\frac{n}{2}(\mathbb{B})}}^{2} d \tau+\int_{0}^{t}\left\|\nabla_{\mathbb{B}} u_{\tau}\right\|_{L_{2}^{\frac{n}{2}(\mathbb{B})}}^{2} d \tau+J(u(t)) \leq J\left(u_{0}\right)<d(\delta), \quad \delta_{1}<\delta<\delta_{2}, \quad 0 \leq t<T_{\max }
$$

we can see that $J\left(u\left(t_{0}\right)\right) \neq d\left(\delta_{0}\right)$. If $I_{\delta_{0}}\left(u\left(t_{0}\right)\right)=0,\left\|\nabla_{\mathbb{B}} u\right\|_{L_{2}^{2}(\mathbb{B})}^{2} \neq 0$, then by the definition of $d(\delta)$, we have $J\left(u\left(t_{0}\right)\right) \geq d\left(\delta_{0}\right)$, which contradicts (3.9).

(2) Let $u(t)$ be a weak solution of problem (1.1) with $0<J\left(u_{0}\right) \leq e<d, I\left(u_{0}\right)<0$. From $J\left(u_{0}\right) \leq e$, $I\left(u_{0}\right)<0$ and Lemma 3.5, it follows $I_{\delta}\left(u_{0}\right)<0$ and $J\left(u_{0}\right)<d(\delta)$. Then $u_{0}(x) \in V_{\delta}$ for $\delta_{1}<\delta<\delta_{2}$. We prove $u(t) \in V_{\delta}$ for $\delta_{1}<\delta<\delta_{2}$ and $0<t<T_{\max }$. Arguing by contradiction, by time continuity of $I(u)$ we suppose that there must exist a $\delta_{0} \in\left(\delta_{1}, \delta_{2}\right)$ and $t_{0} \in\left(0, T_{\text {max }}\right)$ such that $u\left(t_{0}\right) \in \partial V_{\delta_{0}}$, and $I_{\delta_{0}}\left(u\left(t_{0}\right)\right)=0$ or $J\left(u\left(t_{0}\right)\right)=d\left(\delta_{0}\right)$. By $(3.9)$ we can see that $J\left(u\left(t_{0}\right)\right) \neq d\left(\delta_{0}\right)$. Assume $I_{\delta_{0}}\left(u\left(t_{0}\right)\right)=0$ and $t_{0}$ is the first time such that $I_{\delta_{0}}(u(t))=0$, then $I_{\delta_{0}}(u(t))<0$ for $0 \leq t<t_{0}$. By Lemma $3.2(2)$ we have $\left\|\nabla_{\mathbb{B}} u\right\|_{L_{2}^{\frac{n}{2}(\mathbb{B})}}>\gamma\left(\delta_{0}\right)$ for $0 \leq t<t_{0}$. Hence $\left\|\nabla_{\mathbb{B}} u\right\|_{L_{2}^{\frac{n}{2}(\mathbb{B})}} \geq \gamma\left(\delta_{0}\right)$, then $\left\|u\left(t_{0}\right)\right\|_{\mathcal{H}_{2,0}^{1, \frac{n}{2}}(\mathbb{B})} \neq 0$. From $u\left(t_{0}\right) \in \mathcal{N}_{\delta_{0}}$ and $J\left(u\left(t_{0}\right)\right) \neq d\left(\delta_{0}\right)$, we have $J\left(u\left(t_{0}\right)\right)>d\left(\delta_{0}\right)$, which contradicts to (3.9).

To discuss about the invariant of the solutions with negative level energy, we introduce the following results.

Proposition 3. All nontrivial solutions of problem (1.1) with $J\left(u_{0}\right)=0$ belong to

$$
B_{\gamma_{0}}^{c}=\left\{u \in \mathcal{H}_{2,0}^{1, \frac{n}{2}}(\mathbb{B}) \mid\left\|\nabla_{\mathbb{B}} u\right\|_{L_{2}^{\frac{n}{2}}(\mathbb{B})} \geq \gamma_{0}\right\},
$$

where $\gamma_{0}$ is the unique real root of equation

$$
\frac{C_{*}^{p+1}}{p+1} \gamma^{p-1}=\frac{1}{2}
$$

Proof. Let $u(t)$ be any solution of problem (1.1) with $J\left(u_{0}\right)=0, T_{\max }$ be the maximal existence time of $u(t)$. From (3.8), we get $J(u) \leq 0$ for $0 \leq t<T_{\max }$. Hence by

$$
\begin{aligned}
\frac{1}{2}\left\|\nabla_{\mathbb{B}} u\right\|_{L_{2}^{\frac{n}{2}(\mathbb{B})}}^{2} & \leq \frac{1}{p+1} \int_{\mathbb{B}}|u|^{p+1} \frac{d x_{1}}{x_{1}} d x^{\prime} \\
& \leq \frac{1}{p+1} C_{*}^{p+1}\left\|\nabla_{\mathbb{B}} u\right\|_{L_{2}^{\frac{n}{2}}(\mathbb{B})}^{p-1}\left\|\nabla_{\mathbb{B}} u\right\|_{L_{2}^{\frac{n}{2}(\mathbb{B})}}^{2}, 0 \leq t<T_{\max } .
\end{aligned}
$$

we must have either $\left\|\nabla_{\mathbb{B}} u\right\|_{L_{2}^{\frac{n}{2}}(\mathbb{B})}=0$ or $\left\|\nabla_{\mathbb{B}} u\right\|_{L_{2}^{\frac{n}{2}(\mathbb{B})}} \geq \gamma_{0}$. If $\left\|\nabla_{\mathbb{B}} u_{0}\right\|_{L_{2}^{\frac{n}{2}}(\mathbb{B})}=0$, then $\left\|\nabla_{\mathbb{B}} u\right\|_{L_{2}^{\frac{n}{2}}(\mathbb{B})} \equiv 0$ for $0 \leq t<T_{\text {max }}$. Otherwise there exists a $t_{0} \in\left(0, T_{\max }\right)$ such that $0<\left\|\nabla_{\mathbb{B}} u\left(t_{0}\right)\right\|_{L_{2}^{\frac{n}{2}}(\mathbb{B})}<\gamma_{0}$. By a similar argument we can prove that if $\left\|\nabla_{\mathbb{B}} u_{0}\right\|_{L_{2}^{\frac{n}{2}(\mathbb{B})}} \geq \gamma_{0}$, then $\left\|\nabla_{\mathbb{B}} u\right\|_{L_{2}^{\frac{n}{2}(\mathbb{B})}} \geq \gamma_{0}$ for $0<t<T_{\text {max }}$.

Theorem 3.2. Let $u_{0} \in \mathcal{H}_{2,0}^{1, \frac{n}{2}}(\mathbb{B})$. Assume that $J\left(u_{0}\right)<0$ or $J\left(u_{0}\right)=0$ and $\left\|\nabla_{\mathbb{B}} u_{0}\right\|_{L_{2}^{2}(\mathbb{B})} \neq 0$. Then all solutions of problem (1.1) belong to $V_{\delta}$ for $0<\delta<\frac{p+1}{2}$. 
Proof. Let $u(t)$ be any solution of problem (1.1) with $J\left(u_{0}\right)<0$ or $J\left(u_{0}\right)=0$ and $\left\|\nabla_{\mathbb{B}} u_{0}\right\|_{L_{2}^{\frac{n}{2}}(\mathbb{B})} \neq 0$, $T_{\max }$ be the maximal existence time of $u(t)$. The energy inequality gives

$$
a(\delta)\left\|\nabla_{\mathbb{B}} u\right\|_{L_{2}^{\frac{n}{2}(\mathbb{B})}}^{2}+\frac{1}{p+1} I_{\delta}(u)=J(u) \leq J\left(u_{0}\right), \quad 0<\delta<\frac{p+1}{2} .
$$

From (3.11) it follows that if $J\left(u_{0}\right)<0$, then $I_{\delta}(u)<0$ and $J(u)<0<d(\delta)$ for $0<\delta<\frac{p+1}{2}$; if $J\left(u_{0}\right)=0$ and $\left\|\nabla_{\mathbb{B}} u_{0}\right\|_{L_{2}^{\frac{n}{2}}(\mathbb{B})} \neq 0$, then by Proposition 3 we have $\left\|\nabla_{\mathbb{B}} u\right\|_{L_{2}^{\frac{n}{2}}(\mathbb{B})} \geq \gamma_{0}$ for $0 \leq t<T_{\max }$. Again by (3.11) we get $I_{\delta}(u)<0$ and $J(u)<0<d(\delta)$ for $0<\delta<\frac{p+1}{2}$. Hence for above two cases we always have $u(t) \in V_{\delta}$ for $0<\delta<\frac{p+1}{2}, 0 \leq t<T_{\max }$.

Corollary 3.1. Let $u_{0} \in \mathcal{H}_{2,0}^{1, \frac{n}{2}}(\mathbb{B})$. Assume that $J\left(u_{0}\right)<0$ or $J\left(u_{0}\right)=0$ and $\left\|\nabla_{\mathbb{B}} u_{0}\right\|_{L_{2}^{\frac{n}{2}}(\mathbb{B})} \neq 0$. Then all weak solutions of problem (1.1) belong to $\bar{B}_{\frac{p+1}{2}}^{c}$.

Proof. Let $u(t)$ be any weak solution of problem (1.1) with $J\left(u_{0}\right)<0$ or $J\left(u_{0}\right)=0$ and $\left\|\nabla_{\mathbb{B}} u_{0}\right\|_{L_{2}^{\frac{n}{2}}(\mathbb{B})} \neq 0$, $T_{\max }$ be the maximal existence time of $u(t)$. Then Theorem 3.2 gives

$$
u(t) \in V_{\delta} \text { for } 0<\delta<\frac{p+1}{2}, 0 \leq t<T_{\max } .
$$

From this and Lemma 3.2 we get $\left\|\nabla_{\mathbb{B}} u\right\|_{L_{2}^{\frac{n}{2}(\mathbb{B})}}>\gamma(\delta)$ for $0<\delta<\frac{p+1}{2}, 0 \leq t<T_{\max }$. Letting $\delta \rightarrow \frac{p+1}{2}$, we obtain $\left\|\nabla_{\mathbb{B}} u\right\|_{L_{2}^{\frac{n}{2}}(\mathbb{B})} \geq \gamma\left(\frac{p+1}{2}\right)$ for $0 \leq t<T_{\max }$.

Now, we discuss the vacuum isolating to problem $(1.1)$ with $J\left(u_{0}\right)<d$.

Theorem 3.3. Let $e \in(0, d)$. Suppose $\delta_{1}, \delta_{2}$ are the two of $d(\delta)=e$. Then for all weak solutions of problem (1.1) with $J\left(u_{0}\right) \leq e$, there is a vacuum region

$$
U_{e}=\left\{u \in \mathcal{H}_{2,0}^{1, \frac{n}{2}}(\mathbb{B}) \mid I_{\delta}(u)=0,\left\|\nabla_{\mathbb{B}} u\right\|_{L_{2}^{\frac{n}{2}(\mathbb{B})}} \neq 0, \delta_{1}<\delta<\delta_{2}\right\}
$$

such that there is no any weak solution of problem (1.1) in $U_{e}$.

Proof. Let $u(t)$ be any weak solution of problem (1.1) with $J\left(u_{0}\right) \leq e, T_{\text {max }}$ be the maximal existence time of $u(t)$. We only need to prove that if $\left\|\nabla_{\mathbb{B}} u\right\|_{L_{2}^{\frac{n}{2}}(\mathbb{B})} \neq 0$ and $J\left(u_{0}\right) \leq e$, then for all $\delta \in\left(\delta_{1}, \delta_{2}\right)$, $u(t) \notin N_{\delta}$, i.e. $I_{\delta}(u(t)) \neq 0$, for all $t \in\left[0, T_{\max }\right)$.

At first, it is clear that $I_{\delta}\left(u_{0}\right) \neq 0$. Since if $I_{\delta}\left(u_{0}\right)=0$, then $J\left(u_{0}\right) \geq d(\delta)>d\left(\delta_{1}\right)=d\left(\delta_{2}\right)$, which contradicts with $J\left(u_{0}\right) \leq e$.

Suppose there is $t_{1}>0$ s.t. $u\left(t_{1}\right) \in U_{e}$. Namely, there must exist a $\delta_{0} \in\left(\delta_{1}, \delta_{2}\right)$ such that $u\left(t_{1}\right) \in N_{\delta_{0}}$. From (3.8), we get $J\left(u_{0}\right) \geq J\left(u\left(t_{1}\right)\right) \geq d(\delta)>J\left(u_{0}\right)$, which leads to a contradiction.

\section{Low initial ENERGy $J\left(u_{0}\right)<d$}

In this section, we prove a threshold result of global existence and nonexistence of solutions for problem (1.1) with the low initial energy $J\left(u_{0}\right)<d$. 
4.1. Global existence with exponential decay. In this subsection, we establish the global existence of weak solutions for problem (1.1) when $J\left(u_{0}\right)<d$ and $I\left(u_{0}\right)>0$ or $\left\|\nabla_{\mathbb{B}} u_{0}\right\|_{L_{2}^{\frac{n}{2}(\mathbb{B})}}=0$ by using Galerkin approximation technique and potential well theory. Meanwhile, we obtain the asymptotic stability of global solutions.

Theorem 4.1 (Global existence and decay for $J\left(u_{0}\right)<d$ ). Let $u_{0} \in \mathcal{H}_{2,0}^{1, \frac{n}{2}}(\mathbb{B})$. Assume that $J\left(u_{0}\right)<$ $d$ and $I\left(u_{0}\right)>0$ or $\left\|\nabla_{\mathbb{B}} u_{0}\right\|_{L_{2}^{\frac{n}{2}}(\mathbb{B})}=0$. Then problem (1.1) admits a global weak solution $u(t) \in$ $L^{\infty}\left(0, \infty ; \mathcal{H}_{2,0}^{1, \frac{n}{2}}(\mathbb{B})\right)$ with $u_{t} \in L^{2}\left(0, \infty ; \mathcal{H}_{2,0}^{1, \frac{n}{2}}(\mathbb{B})\right)$. Moreover $u(t) \in W$ for $0 \leq t<\infty$, and there exist constants $C>0$ such that

$$
\|u\|_{L_{2}^{\frac{n}{2}(\mathbb{B})}}^{2}+\left\|\nabla_{\mathbb{B}} u\right\|_{L_{2}^{\frac{n}{2}}(\mathbb{B})}^{2} \leq\left(\left\|u_{0}\right\|_{L_{2}^{\frac{n}{2}(\mathbb{B})}}^{2}+\left\|\nabla_{\mathbb{B}} u_{0}\right\|_{L_{2}^{\frac{n}{2}}(\mathbb{B})}^{2}\right) e^{-C t} .
$$

Proof. We divide the proof into two steps.

Step 1 Proof of global existence.

Let $\left\{\omega_{j}(x)\right\}$ be a system of base functions in $\mathcal{H}_{2,0}^{1, \frac{n}{2}}(\mathbb{B})$. Now we construct the following approximate solutions $u_{m}(t, x)$ of problem (1.1):

$$
u_{m}(t, x)=\sum_{j=1}^{m} g_{j m}(t) \omega_{j}(x), \quad m=1,2, \cdots,
$$

which satisfies

$$
\begin{gathered}
\left(u_{m t}, \omega_{s}\right)_{2}+\left(\nabla_{\mathbb{B}} u_{m}, \nabla_{\mathbb{B}} \omega_{s}\right)_{2}+\left(\nabla_{\mathbb{B}} u_{m t}, \nabla_{\mathbb{B}} \omega_{s}\right)_{2}=\left(\left|u_{m}\right|^{p-1} u_{m}, \omega_{s}\right)_{2}, \quad s=1,2, \cdots, \\
u_{m}(x, 0)=\sum_{j=1}^{m} a_{j m} \omega_{j}(x) \rightarrow u_{0}(x) \text { in } \mathcal{H}_{2,0}^{1, \frac{n}{2}}(\mathbb{B}) .
\end{gathered}
$$

Multiplying (4.1) by $g_{s m}^{\prime}(t)$, summing for $s$, and integrating with respect to $t$ from 0 to $t$, we have

$$
\int_{0}^{t}\left\|u_{m \tau}\right\|_{L_{2}^{\frac{n}{2}(\mathbb{B})}}^{2} d \tau+\int_{0}^{t}\left\|\nabla_{\mathbb{B}} u_{m \tau}\right\|_{L_{2}^{\frac{n}{2}}(\mathbb{B})}^{2} d \tau+J\left(u_{m}\right) \leq J\left(u_{m}(0)\right), \quad 0 \leq t<\infty .
$$

By (4.2) we can get $J\left(u_{m}(0)\right) \rightarrow J\left(u_{0}\right)$, then for sufficiently large $m$, we have

$$
\int_{0}^{t}\left\|u_{m \tau}\right\|_{L_{2}^{\frac{n}{2}}(\mathbb{B})}^{2} d \tau+\int_{0}^{t}\left\|\nabla_{\mathbb{B}} u_{m \tau}\right\|_{L_{2}^{\frac{n}{2}}(\mathbb{B})}^{2} d \tau+J\left(u_{m}\right)<d, \quad 0 \leq t<\infty .
$$

From (4.3) and the proof of Theorem 3.1, we can get $u_{m}(t) \in W$ for $0 \leq t<\infty$ and sufficiently large $m$. Hence, by (4.3) and

$$
J\left(u_{m}\right)=\frac{p-1}{2(p+1)}\left\|\nabla_{\mathbb{B}} u_{m}\right\|_{L_{2}^{\frac{n}{2}}(\mathbb{B})}^{2}+\frac{1}{p+1} I\left(u_{m}\right),
$$

we obtain

$$
\int_{0}^{t}\left\|u_{m \tau}\right\|_{L_{2}^{\frac{n}{2}}(\mathbb{B})}^{2} d \tau+\int_{0}^{t}\left\|\nabla_{\mathbb{B}} u_{m \tau}\right\|_{L_{2}^{\frac{n}{2}}(\mathbb{B})}^{2} d \tau+\frac{p-1}{2(p+1)}\left\|\nabla_{\mathbb{B}} u_{m}\right\|_{L_{2}^{\frac{n}{2}}(\mathbb{B})}^{2}<d, \quad 0 \leq t<\infty,
$$

for sufficiently large $m$, which yields

$$
\left\|\nabla_{\mathbb{B}} u_{m}\right\|_{L_{2}^{\frac{n}{2}}(\mathbb{B})}^{2}<\frac{2(p+1)}{p-1} d, \quad 0 \leq t<\infty,
$$




$$
\begin{gathered}
\int_{0}^{t}\left\|\nabla_{\mathbb{B}} u_{m \tau}\right\|_{L_{2}^{\frac{n}{2}(\mathbb{B})}}^{2} d \tau<d, \quad 0 \leq t<\infty, \\
\left.\left.\int_{\mathbb{B}}|| u_{m}\right|^{p-1} u_{m}\right|^{\frac{p+1}{p}} \frac{d x_{1}}{x_{1}} d x^{\prime}=\int_{\mathbb{B}}\left|u_{m}\right|^{p+1} \frac{d x_{1}}{x_{1}} d x^{\prime}=\left\|u_{m}\right\|_{L_{p+1}^{p+1}(\mathbb{B})}^{p+1} \\
\leq C_{*}^{p+1}\left\|\nabla_{\mathbb{B}} u_{m}\right\|_{L_{2}^{\frac{n}{2}}(\mathbb{B})}^{p+1}<C^{p+1}\left(\frac{2(p+1)}{p-1} d\right)^{\frac{p+1}{2}} .
\end{gathered}
$$

Therefore, there exist a $u$ and a subsequence $\left\{u_{v}\right\}$ such that

$$
\begin{aligned}
& u_{v} \rightarrow u \text { in } L^{\infty}\left(0, \infty ; \mathcal{H}_{2,0}^{1, \frac{n}{2}}(\mathbb{B})\right) \text { weakly star and a.e. in } \operatorname{int} \mathbb{B} \times[0, \infty), \\
& u_{v t} \rightarrow u_{t} \text { in } L^{2}\left(0, \infty ; \mathcal{H}_{2,0}^{1, \frac{n}{2}}(\mathbb{B})\right) \text { weakly star, } \\
& u_{v}^{p} \rightarrow u^{p} \text { in } L^{\infty}\left(0, \infty ; L_{\frac{p+1}{p}}^{\frac{p n}{p+1}}(\mathbb{B})\right) \text { weakly star and a.e. in } \operatorname{int} \mathbb{B} \times[0, \infty) .
\end{aligned}
$$

In (4.1), we fixed $s$, letting $m=v \rightarrow \infty$. Then, we get

$$
\left(u_{t}, \omega_{s}\right)_{2}+\left(\nabla_{\mathbb{B}} u, \nabla_{\mathbb{B}} \omega_{s}\right)_{2}+\left(\nabla_{\mathbb{B}} u_{t}, \nabla_{\mathbb{B}} \omega_{s}\right)_{2}=\left(|u|^{p-1} u, \omega_{s}\right)
$$

and

$$
\left(u_{t}, v\right)_{2}+\left(\nabla_{\mathbb{B}} u, \nabla_{\mathbb{B}} v\right)_{2}+\left(\nabla_{\mathbb{B}} u_{t}, \nabla_{\mathbb{B}} v\right)_{2}=\left(|u|^{p-1} u, v\right), \quad \forall v \in \mathcal{H}_{2,0}^{1, \frac{n}{2}}(\Omega), t \in(0, T) .
$$

From (4.2) we obtain $u(x, 0)=u_{0}(x)$ in $\mathcal{H}_{2,0}^{1, \frac{n}{2}}(\mathbb{B}), t \in(0, T)$. By density we obtain $u \in$ $L^{\infty}\left(0, \infty ; \mathcal{H}_{2,0}^{1, \frac{n}{2}}(\mathbb{B})\right)$ with $u_{t} \in L^{2}\left(0, \infty ; \mathcal{H}_{2,0}^{1, \frac{n}{2}}(\mathbb{B})\right)$ is a global weak solution of problem (1.1). It is obvious that $u(t) \in W$ for $0 \leq t<\infty$.

Step 2 Proof of decay.

Taking $v=u$ in (3.7), we get

$$
\frac{1}{2} \frac{d}{d t}\left(\|u\|_{L_{2}^{\frac{n}{2}(\mathbb{B})}}^{2}+\left\|\nabla_{\mathbb{B}} u\right\|_{L_{2}^{\frac{n}{2}(\mathbb{B})}}^{2}\right)=-\left\|\nabla_{\mathbb{B}} u\right\|_{L_{2}^{\frac{n}{2}(\mathbb{B})}}^{2}+\|u\|_{L_{p+1}^{\frac{n}{p+1}(\mathbb{B})}}^{p+1}=-I(u) .
$$

From Theorem 3.1 we have that $u(x, t) \in W_{\delta}$ for $\delta_{1}<\delta<\delta_{2}$ and $0<t<\infty$ under the condition $J\left(u_{0}\right)<d$ and $I\left(u_{0}\right)>0$ or $\left\|\nabla_{\mathbb{B}} u_{0}\right\|_{L_{2}^{\frac{n}{2}}(\mathbb{B})}=0$. Thus, by Lemma 3.5, we have $I\left(\delta_{1}\right) \geq 0$ for $0<t<\infty$. Therefore,

$$
\begin{aligned}
\frac{1}{2} \frac{d}{d t}\left(\|u\|_{L_{2}^{\frac{n}{2}(\mathbb{B})}}^{2}+\left\|\nabla_{\mathbb{B}} u\right\|_{L_{2}^{\frac{n}{2}}(\mathbb{B})}^{2}\right)=-I(u) & =\left(\delta_{1}-1\right)\left\|\nabla_{\mathbb{B}} u\right\|_{L_{2}^{\frac{n}{2}}(\mathbb{B})}^{2}-I_{\delta_{1}}(u) \\
& \leq \frac{1}{C_{*}^{2}+1}\left(\delta_{1}-1\right)\left(\|u\|_{L_{2}^{\frac{n}{2}}(\mathbb{B})}^{2}+\left\|\nabla_{\mathbb{B}} u\right\|_{L_{2}^{\frac{n}{2}}(\mathbb{B})}^{2}\right) .
\end{aligned}
$$

Consequently,

$$
\|u\|_{L_{2}^{\frac{n}{2}(\mathbb{B})}}^{2}+\left\|\nabla_{\mathbb{B}} u\right\|_{L_{2}^{\frac{n}{2}(\mathbb{B})}}^{2} \leq\left(\left\|u_{0}\right\|_{L_{2}^{\frac{n}{2}(\mathbb{B})}}^{2}+\left\|\nabla_{\mathbb{B}} u_{0}\right\|_{L_{2}^{\frac{n}{2}(\mathbb{B})}}^{2}\right) e^{-C t},
$$

where $C=2 \frac{1-\delta_{1}}{C_{*}^{2}+1}$.

The proof is complete.

Corollary 4.1. In Theorem 4.1, if the assumptions " $J\left(u_{0}\right)<d, I\left(u_{0}\right)>0$ " is replaced by " $0<$ $J\left(u_{0}\right)<d, I_{\delta_{2}}\left(u_{0}\right)>0$ ", where $\left(\delta_{1}, \delta_{2}\right)$ is the maximal interval including $\delta=1$ such that $d_{\delta}>J\left(u_{0}\right)$ 
for $\delta \in\left(\delta_{1}, \delta_{2}\right)$, then problem (1.1) admits a global weak solution $u(t) \in L^{\infty}\left(0, \infty ; \mathcal{H}_{2,0}^{1, \frac{n}{2}}(\mathbb{B})\right)$ with $u_{t} \in L^{2}\left(0, \infty ; \mathcal{H}_{2,0}^{1, \frac{n}{2}}(\mathbb{B})\right)$ and $u(t) \in W_{\delta}$, for $0 \leq t<\infty$.

Proof. Making use of Lemma 3.5, we obtain from $0<J\left(u_{0}\right)<d, I_{\delta_{2}}\left(u_{0}\right)>0$ that $I_{\delta}\left(u_{0}\right)>0$ for all $\delta \in\left(\delta_{1}, \delta_{2}\right)$. Repeating the arguments of Theorem 4.1 for $\delta_{1}<\delta<\delta_{2}$, then the conclusion of Corollary 4.1 holds.

Corollary 4.2. In Corollary 4.1, if the assumptions " $I_{\delta_{2}}\left(u_{0}\right)>0$ or $\left\|\nabla_{\mathbb{B}} u_{0}\right\|_{L_{2}^{\frac{n}{2}}(\mathbb{B})}=0$ " is replaced by $\left\|\nabla_{\mathbb{B}} u\right\|_{L_{2}^{\frac{n}{2}}(\mathbb{B})}<\gamma(\delta)$, then problem (1.1) admits a global weak solution $u(t) \in L^{\infty}\left(0, \infty ; \mathcal{H}_{2,0}^{1, \frac{n}{2}}(\mathbb{B})\right)$ with $u_{t} \in L^{2}\left(0, \infty ; \mathcal{H}_{2,0}^{1, \frac{n}{2}}(\mathbb{B})\right)$ and satisfies

$$
\left\|\nabla_{\mathbb{B}} u\right\|_{L_{2}^{\frac{n}{2}(\mathbb{B})}}^{2}<\frac{d(\delta)}{a(\delta)}, \quad \text { for } \delta_{1}<\delta<\min \left\{\delta_{2}, \frac{p+1}{2}\right\}, 0 \leq t<\infty .
$$

In particular, we have

$$
\left\|\nabla_{\mathbb{B}} u\right\|_{L_{2}^{\frac{n}{2}(\mathbb{B})}}^{2} \leq \frac{d\left(\delta_{1}\right)}{a\left(\delta_{1}\right)} \quad \text { for } 0 \leq t<\infty .
$$

Proof. $\left\|\nabla_{\mathbb{B}} u\right\|_{L_{2}^{\frac{n}{2}(\mathbb{B})}}<\gamma(\delta)$ gives $I_{\delta_{2}}\left(u_{0}\right)>0$ or $\left\|\nabla_{\mathbb{B}} u_{0}\right\|_{L_{2}^{\frac{n}{2}(\mathbb{B})}}=0$. Hence, from Corollary 4.1, it follows that problem $(1.1)$ admits a global weak solution $u(t) \in L^{\infty}\left(0, \infty ; \mathcal{H}_{2,0}^{1, \frac{n}{2}}(\mathbb{B})\right)$ with $u_{t} \in L^{2}\left(0, \infty ; \mathcal{H}_{2,0}^{1, \frac{n}{2}}(\mathbb{B})\right)$ and $u(t) \in W_{\delta}$, for $0 \leq t<\infty$. Moreover, from Lemma 3.4, we can deduce that (4.8) holds. Letting $\delta \rightarrow \delta_{1}$, the conclusion (4.9) is also obtained.

4.2. Finite time blow-up of solution. In this subsection, we establish finite time blow-up of solution for problem (1.1) when $J\left(u_{0}\right)<d$ and $I\left(u_{0}\right)<0$ by using the concavity argument (see $[13,14,15]$ ) and properties of a family of potential wells. Furthermore, by making use of a differential inequality technique (see [18]) we determine a lower bound on blow-up time for certain solutions of problem (1.1) if blow-up occurs.

We need the following lemmas to prove finite time blow-up with $J\left(u_{0}\right)<d$.

Lemma 4.1. Let $u_{0} \in \mathcal{H}_{2,0}^{1, \frac{n}{2}}(\mathbb{B})$. Suppose $J\left(u_{0}\right)<d$ and $I\left(u_{0}\right)<0$, then we have

$$
d<\frac{p-1}{2(p+1)}\left\|\nabla_{\mathbb{B}} u\right\|_{L_{2}^{\frac{n}{2}(\mathbb{B})}}^{2}
$$

for all $t \in\left[0, T_{\max }\right)$, where $T_{\max }$ is the maximal existence time.

Proof. We first prove $I(u)<0$ for $t>0$. If it is false, we must be allowed to choose a $t_{0}>0$ such that $I\left(u\left(t_{0}\right)\right)=0$ and $I(u)<0$ for $0 \leq t<t_{0}$. From Lemma $3.2(2)$, we have $\left\|\nabla_{\mathbb{B}} u(t)\right\|_{L_{2}^{\frac{n}{2}}(\mathbb{B})}>\gamma(1)$ for $0 \leq t<t_{0},\left\|\nabla_{\mathbb{B}} u\left(t_{0}\right)\right\|_{L_{2}^{\frac{n}{2}}(\mathbb{B})}^{2} \geq \gamma(1)$ and $J\left(u\left(t_{0}\right)\right) \geq d$, which contradicts to (3.8). Since

$$
J(\lambda u)=\frac{\lambda^{2}}{2} \int_{\mathbb{B}}\left|\nabla_{\mathbb{B}} u\right|^{2} \frac{d x_{1}}{x_{1}} d x^{\prime}-\frac{\lambda^{p+1}}{p+1} \int_{\mathbb{B}}|u|^{p+1} \frac{d x_{1}}{x_{1}} d x^{\prime},
$$

we get

$$
\frac{d}{d \lambda} J(\lambda u)=\lambda \int_{\mathbb{B}}\left|\nabla_{\mathbb{B}} u\right|^{2} \frac{d x_{1}}{x_{1}} d x^{\prime}-\lambda^{p} \int_{\mathbb{B}}|u|^{p+1} \frac{d x_{1}}{x_{1}} d x^{\prime}
$$


and

$$
\frac{d^{2}}{d \lambda^{2}} J(\lambda u)=\int_{\mathbb{B}}\left|\nabla_{\mathbb{B}} u\right|^{2} \frac{d x_{1}}{x_{1}} d x^{\prime}-p \lambda^{p-1} \int_{\mathbb{B}}|u|^{p+1} \frac{d x_{1}}{x_{1}} d x^{\prime}
$$

Let $\frac{d}{d \lambda} J(\lambda u)=0$, which implies

$$
\overline{\lambda_{1}}=0, \quad \overline{\lambda_{2}}=\left(\frac{\int_{\mathbb{B}}\left|\nabla_{\mathbb{B}} u\right|^{2} \frac{d x_{1}}{x_{1}} d x^{\prime}}{\int_{\mathbb{B}}|u|^{p+1} \frac{d x_{1}}{x_{1}} d x^{\prime}}\right)^{\frac{1}{p-1}} .
$$

An elementary calculation shows

$$
\frac{d^{2}}{d \lambda^{2}} J\left(\overline{\lambda_{1}} u\right)>0 \quad \text { and } \quad \frac{d^{2}}{d \lambda^{2}} J\left(\overline{\lambda_{2}} u\right)<0 .
$$

So we have

$$
\sup _{\lambda \geq 0} J(\lambda u)=J\left(\overline{\lambda_{2}} u\right)=\frac{p-1}{2(p+1)} \frac{\left(\int_{\mathbb{B}}\left|\nabla_{\mathbb{B}} u\right|^{2} \frac{d x_{1}}{x_{1}} d x^{\prime}\right)^{\frac{p+1}{p-1}}}{\left(\int_{\mathbb{B}}|u|^{p+1} \frac{d x_{1}}{x_{1}} d x^{\prime}\right)^{\frac{2}{p-1}}} .
$$

By $I(u)<0$, we have

$$
\begin{aligned}
d & \leq \sup _{\lambda \geq 0} J(\lambda u)=J\left(\bar{\lambda}_{2} u\right)=\frac{p-1}{2(p+1)} \frac{\left(\int_{\mathbb{B}}\left|\nabla_{\mathbb{B}} u\right|^{2} \frac{d x_{1}}{x_{1}} d x^{\prime}\right)^{\frac{p+1}{p-1}}}{\left(\int_{\mathbb{B}}|u|^{p+1} \frac{d x_{1}}{x_{1}} d x^{\prime}\right)^{\frac{2}{p-1}}} \\
& <\frac{p-1}{2(p+1)}\left\|\nabla_{\mathbb{B}} u\right\|_{L_{2}^{\frac{n}{2}}(\mathbb{B})}^{2} .
\end{aligned}
$$

Lemma $4.2([12])$. Let $L(t)$ be a positive, twice differentiable function, which satisfies, for $t>0$, inequality

$$
L(t) L^{\prime \prime}(t)-(1+\alpha) L^{\prime}(t)^{2} \geq 0
$$

with some $\alpha>0$. If $L(0)>0$ and $L^{\prime}(0)>0$, then there exists a time $T^{*} \leq \frac{L(0)}{\left[\alpha L^{\prime}(0)\right]}$ such that $\lim _{t \rightarrow T^{*-}} L(t)=\infty$.

Theorem 4.2. Let $u_{0} \in \mathcal{H}_{2,0}^{1, \frac{n}{2}}(\mathbb{B})$. Suppose $J\left(u_{0}\right)<d$ and $I\left(u_{0}\right)<0$. Then the existence time of the weak solution for problem (1.1) is finite, i.e., the maximal existence time $T_{\text {max }}$ is finite and

$$
\lim _{t \rightarrow T_{\text {max }}^{-}} \int_{0}^{t}\left(\|u\|_{L_{2}^{\frac{n}{2}}(\mathbb{B})}^{2}+\left\|\nabla_{\mathbb{B}} u\right\|_{L_{2}^{\frac{n}{2}}(\mathbb{B})}^{2}\right) d \tau=+\infty .
$$

Moreover, we have

$$
T_{\max } \leq \frac{b T_{0}^{2}}{b(p-1) T_{0}-\left(\left\|u_{0}\right\|_{L_{2}^{\frac{n}{2}}(\mathbb{B})}^{2}+\left\|\nabla_{\mathbb{B}} u_{0}\right\|_{L_{2}^{\frac{n}{2}(\mathbb{B})}}^{2}\right)}
$$

where $b$ and $T_{0}$ to be chosen later. 
Proof. Let $u(t)$ be any weak solution of problem (1.1) with $J\left(u_{0}\right)<d$ and $I\left(u_{0}\right)<0$. By contradiction, we suppose that $u(t)$ is global, then $T_{\max }=\infty$. For any $T>0$ and for all $t \in[0, T]$, we define

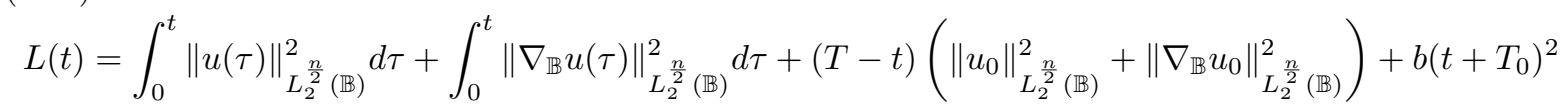

where $b$ and $T_{0}$ are positive constants which will be specified later. Furthermore,

$$
\begin{aligned}
L^{\prime}(t) & =\|u(t)\|_{L_{2}^{\frac{n}{2}}(\mathbb{B})}^{2}+\left\|\nabla_{\mathbb{B}} u(t)\right\|_{L_{2}^{\frac{n}{2}}(\mathbb{B})}^{2}-\left(\left\|u_{0}\right\|_{L_{2}^{\frac{n}{2}}(\mathbb{B})}^{2}+\left\|\nabla_{\mathbb{B}} u_{0}\right\|_{L_{2}^{\frac{n}{2}}(\mathbb{B})}^{2}\right)+2 b\left(t+T_{0}\right) \\
& =2 \int_{0}^{t}\left(u(\tau), u_{\tau}(\tau)\right)_{2} d \tau+2 \int_{0}^{t}\left(\nabla_{\mathbb{B}} u(\tau), \nabla_{\mathbb{B}} u_{\tau}(\tau)\right)_{2} d \tau+2 b\left(t+T_{0}\right)
\end{aligned}
$$

and, consequently,

Therefore, we get

$$
L^{\prime \prime}(t)=2 \int_{\mathbb{B}} u(t) u_{t}(t) \frac{d x_{1}}{x_{1}} d x^{\prime}+2 \int_{\mathbb{B}} \nabla_{\mathbb{B}} u(t) \nabla_{\mathbb{B}} u_{t}(t) \frac{d x_{1}}{x_{1}} d x^{\prime}+2 b
$$

$$
\begin{aligned}
& L(t) L^{\prime \prime}(t)-\frac{p+1}{2} L^{\prime}(t)^{2} \\
= & L(t) L^{\prime \prime}(t)+2(p+1)\left[\eta(t)-\left(L(t)-(T-t)\left(\left\|u_{0}\right\|_{L_{2}^{\frac{n}{2}}(\mathbb{B})}^{2}+\left\|\nabla_{\mathbb{B}} u_{0}\right\|_{L_{2}^{\frac{n}{2}}(\mathbb{B})}^{2}\right)\right)\right]\left(\int_{0}^{t}\left\|u_{\tau}(\tau)\right\|_{L_{2}^{\frac{n}{2}}(\mathbb{B})}^{2} d \tau\right. \\
& \left.+\int_{0}^{t}\left\|\nabla_{\mathbb{B}} u_{\tau}(\tau)\right\|_{L_{2}^{\frac{n}{2}}(\mathbb{B})}^{2} d \tau+b\right)
\end{aligned}
$$

where $\eta:[0, T] \rightarrow \mathbb{R}_{+}$is the function defined by

$$
\begin{array}{r}
\eta=\left(\int_{0}^{t}\|u(\tau)\|_{L_{2}^{\frac{n}{2}}(\mathbb{B})}^{2} d \tau+\int_{0}^{t}\left\|\nabla_{\mathbb{B}} u(\tau)\right\|_{L_{2}^{\frac{n}{2}}(\mathbb{B})}^{2} d \tau+b\right)\left(\int_{0}^{t}\left\|u_{\tau}(\tau)\right\|_{L_{2}^{\frac{n}{2}}(\mathbb{B})}^{2} d \tau+\int_{0}^{t}\left\|\nabla_{\mathbb{B}} u_{\tau}(\tau)\right\|_{L_{2}^{\frac{n}{2}}(\mathbb{B})}^{2} d \tau\right. \\
\left.+b\left(t+T_{0}\right)^{2}\right)-\left(\int_{0}^{t}\left(u(\tau), u_{\tau}(\tau)\right)_{2} d \tau+\int_{0}^{t}\left(\nabla_{\mathbb{B}} u(\tau), \nabla_{\mathbb{B}} u_{\tau}(\tau)\right)_{2} d \tau+b\left(t+T_{0}\right)\right)^{2} \geq 0
\end{array}
$$

As a consequence, we read the following differential inequality

$$
\begin{aligned}
L(t) L^{\prime \prime}(t)-\frac{p+1}{2} L^{\prime}(t)^{2} & \geq L(t) L^{\prime \prime}(t)-2(p+1) L(t)\left(\int_{0}^{t}\left\|u_{\tau}(\tau)\right\|_{L_{2}^{\frac{n}{2}}(\mathbb{B})}^{2} d \tau+\int_{0}^{t}\left\|\nabla_{\mathbb{B}} u_{\tau}(\tau)\right\|_{L_{2}^{\frac{n}{2}}(\mathbb{B})}^{2} d \tau+b\right) \\
& =L(t) \xi(t),
\end{aligned}
$$

for almost every $t \in[0, T]$, where $\xi:[0, T] \rightarrow \mathbb{R}_{+}$is the map defined by

$$
\begin{aligned}
\xi(t)= & 2 \int_{\mathbb{B}} u(t) u_{t}(t) \frac{d x_{1}}{x_{1}} d x^{\prime}+2 \int_{\mathbb{B}} \nabla_{\mathbb{B}} u(t) \nabla_{\mathbb{B}} u_{t}(t) \frac{d x_{1}}{x_{1}} d x^{\prime}-2(p+1) \int_{0}^{t}\left\|u_{\tau}(\tau)\right\|_{L_{2}^{\frac{n}{2}(\mathbb{B})}}^{2} d \tau \\
& -2(p+1) \int_{0}^{t}\left\|\nabla_{\mathbb{B}} u_{\tau}(\tau)\right\|_{L_{2}^{\frac{n}{2}(\mathbb{B})}}^{2} d \tau-2 p b .
\end{aligned}
$$

By (1.1) and (3.8), we have

$$
\begin{aligned}
\xi(t)= & 2 \int_{\mathbb{B}}\left(u^{p+1}-\left\|\nabla_{\mathbb{B}} u\right\|^{2}\right) \frac{d x_{1}}{x_{1}} d x^{\prime}-2(p+1) \int_{0}^{t}\left\|u_{\tau}(\tau)\right\|_{L_{2}^{\frac{n}{2}}(\mathbb{B})}^{2} d \tau \\
& -2(p+1) \int_{0}^{t}\left\|\nabla_{\mathbb{B}} u_{\tau}(\tau)\right\|_{L_{2}^{\frac{n}{2}}(\mathbb{B})}^{2} d \tau-2 p b \\
\geq & 2 \int_{\mathbb{B}}\left(u^{p+1}-\left\|\nabla_{\mathbb{B}} u\right\|^{2}\right) \frac{d x_{1}}{x_{1}} d x^{\prime}-2(p+1) J\left(u_{0}\right)+2(p+1) J(u(t))-2 p b . \\
\geq & (p-1)\left\|\nabla_{\mathbb{B}} u\right\|_{L_{2}^{\frac{n}{2}}(\mathbb{B})}^{2}-2(p+1) d-2 p b .
\end{aligned}
$$


By Lemma 4.1, we can get $d<\frac{p-1}{2(p+1)}\left\|\nabla_{\mathbb{B}} u\right\|_{L_{2}^{\frac{n}{2}}(\mathbb{B})}^{2}$. Choosing $b$ small enough such that

$$
b \leq \frac{(p-1)\left\|\nabla_{\mathbb{B}} u\right\|_{L_{2}^{\frac{n}{2}(\mathbb{B})}}^{2}-2(p+1) d}{2 p},
$$

we have

which implies

$$
\xi(t) \geq(p-1)\left\|\nabla_{\mathbb{B}} u\right\|_{L_{2}^{\frac{n}{2}}(\mathbb{B})}^{2}-2(p+1) d-2 p b \geq 0,
$$

$$
L(t) L^{\prime \prime}(t)-\frac{p+1}{2} L^{\prime}(t)^{2} \geq 0 .
$$

By (4.14) and (4.15), we have $L(0)>0$ and $L^{\prime}(0)>0$. We then choose $T_{0}$ sufficiently large such that

$$
b(p-1) T_{0}-\left(\left\|u_{0}\right\|_{L_{2}^{\frac{n}{2}(\mathbb{B})}}^{2}+\left\|\nabla_{\mathbb{B}} u_{0}\right\|_{L_{2}^{\frac{n}{2}(\mathbb{B})}}^{2}\right)>0 .
$$

Combining Lemma 4.2, we then obtain that $L(t)$ blows up in finite time

$$
T^{*} \leq \frac{b T_{0}^{2}}{b(p-1) T_{0}-\left(\left\|u_{0}\right\|_{L_{2}^{\frac{n}{2}(\mathbb{B})}}^{2}+\left\|\nabla_{\mathbb{B}} u_{0}\right\|_{L_{2}^{\frac{n}{2}(\mathbb{B})}}^{2}\right)} .
$$

In view of the arbitrariness of time $T$, we may choose $T$ such that

$$
T \geq \frac{b T_{0}^{2}}{b(p-1) T_{0}-\left(\left\|u_{0}\right\|_{L_{2}^{\frac{n}{2}(\mathbb{B})}}^{2}+\left\|\nabla_{\mathbb{B}} u_{0}\right\|_{L_{2}^{\frac{n}{2}(\mathbb{B})}}^{2}\right)} .
$$

Thus, we see from (4.14) that there exists a time $T^{*} \in(0, T]$ such that

$$
\lim _{t \rightarrow T^{*-}} \int_{0}^{t}\left(\|u\|_{L_{2}^{\frac{n}{2}(\mathbb{B})}}^{2}+\left\|\nabla_{\mathbb{B}} u\right\|_{L_{2}^{\frac{n}{2}(\mathbb{B})}}^{2}\right) d \tau=+\infty,
$$

which contradicts $T_{\max }=\infty$. Hence, the solution of the problem (1.1) blows up in finite time.

Theorem 4.3. Under the assumption of Theorem 4.2, then the solution $u(x, t)$ of problem (1.1) blows up at $t=T_{\max }$ in $\mathcal{H}_{2,0}^{1, \frac{n}{2}}(\mathbb{B})$ with $\lim _{t \rightarrow T_{\text {max }}^{-}} \int_{0}^{t}\|u\|_{L_{2}^{\frac{n}{2}}(\mathbb{B})}^{2}+\left\|\nabla_{\mathbb{B}} u\right\|_{L_{2}^{\frac{n}{2}}(\mathbb{B})}^{2} d \tau=+\infty$. Moreover, we have

$$
T_{\max } \geq \frac{\left(\left\|u_{0}\right\|_{L_{2}^{\frac{n}{2}}(\mathbb{B})}^{2}+\left\|\nabla_{\mathbb{B}} u_{0}\right\|_{L_{2}^{\frac{n}{2}(\mathbb{B})}}^{2}\right)^{-\frac{p-1}{2}}}{(p-1) C_{*}^{p+1}} .
$$

Proof. First, from Theorem 4.2, we know that the solution $u(x, t)$ of problem (1.1) blows up in finite time $T_{\max }$. Now, we estimate the lower bound for blow-up time $T_{\max }$.

We define

$$
\varphi(t)=\|u\|_{L_{2}^{\frac{n}{2}(\mathbb{B})}}^{2}+\left\|\nabla_{\mathbb{B}} u\right\|_{L_{2}^{\frac{n}{2}}(\mathbb{B})}^{2} .
$$

Multiplying $u(x, t)$ on two sides of equation (1.1), and integrating by part, we have

$$
\int_{\mathbb{B}} u u_{t} \frac{d x_{1}}{x_{1}} d x^{\prime}+\int_{\mathbb{B}}\left|\nabla_{\mathbb{B}} u_{t}\right|\left|\nabla_{\mathbb{B}} u\right| \frac{d x_{1}}{x_{1}} d x^{\prime}=-\int_{\mathbb{B}}\left|\nabla_{\mathbb{B}} u\right|^{2} \frac{d x_{1}}{x_{1}} d x^{\prime}+\int_{\mathbb{B}}|u|^{p+1} \frac{d x_{1}}{x_{1}} d x^{\prime} .
$$

Then by direct computation and (4.20), we have

$$
\varphi^{\prime}(t)=-2 \int_{\mathbb{B}}\left|\nabla_{\mathbb{B}} u\right|^{2} \frac{d x_{1}}{x_{1}} d x^{\prime}+2 \int_{\mathbb{B}}|u|^{p+1} \frac{d x_{1}}{x_{1}} d x^{\prime},
$$


which implies

$$
\varphi^{\prime}(t) \leq 2 C_{*}^{p+1}(\varphi(t))^{\frac{p+1}{2}}
$$

If there exists $t_{0} \in\left[0, T_{\max }\right)$ such that $\varphi\left(t_{0}\right)=0$, then we can obtain $\varphi\left(T_{\max }\right)=0$, which contradicts with the fact that $u(x, t)$ blows up at $T_{\max }$ in $H_{2,0}^{1, \frac{n}{2}}(\mathbb{B})$-norm. So we see

$$
\varphi(t)>0
$$

and

$$
\frac{\varphi^{\prime}(t)}{(\varphi(t))^{\frac{p+1}{2}}} \leq 2 C_{*}^{p+1} .
$$

Integrating the inequality (4.22) from 0 to $t$, we have

$$
(\varphi(0))^{-\frac{p-1}{2}}-(\varphi(t))^{-\frac{p-1}{2}} \leq(p-1) C_{*}^{p-1} t .
$$

So letting $t \rightarrow T_{\max }$ in $(4.23)$, we can conclude that

$$
T_{\max } \geq \frac{\left(\left\|u_{0}\right\|_{L_{2}^{\frac{n}{2}}(\mathbb{B})}^{2}+\left\|\nabla_{\mathbb{B}} u_{0}\right\|_{L_{2}^{\frac{n}{2}(\mathbb{B})}}^{2}\right)^{-\frac{p-1}{2}}}{(p-1) C_{*}^{p+1}} .
$$

Remark 1. Noting that from

$$
\frac{p-1}{2(p+1)}\left\|\nabla_{\mathbb{B}} u_{0}\right\|_{L_{2}^{\frac{n}{2}}(\mathbb{B})}^{2}+\frac{1}{p+1} I\left(u_{0}\right)=J\left(u_{0}\right),
$$

we see that if $J\left(u_{0}\right)<0$, then $I\left(u_{0}\right) \geq 0$ is impossible. If $J\left(u_{0}\right)=0$, then either $I\left(u_{0}\right)>0$ or $I\left(u_{0}\right)=0$ with $\left\|\nabla_{\mathbb{B}} u_{0}\right\|_{L_{2}^{\frac{n}{2}}(\mathbb{B})}^{2} \neq 0$ is impossible. If $0<J\left(u_{0}\right)<d$, it follows from the definition of $d$ that $I\left(u_{0}\right)=0$ with $\left\|\nabla_{\mathbb{B}} u_{0}\right\|_{L_{2}^{\frac{n}{2}}}^{2} \neq 0$ is impossible. Thus, all possible cases already have been considered in Theorems 4.1 and 4.2.

From the discussion above, a threshold result of global existence and nonexistence of solutions for problem (1.1) has been obtained as follows.

Corollary 4.3. Assume that $u_{0} \in \mathcal{H}_{2,0}^{1, \frac{n}{2}}(\mathbb{B})$ and $J\left(u_{0}\right)<d$. Then problem (1.1) admits a global weak solution provided $I\left(u_{0}\right)>0$ or $\left\|\nabla_{\mathbb{B}} u_{0}\right\|_{L_{2}^{\frac{n}{2}}(\mathbb{B})}^{2}=0 ;$ problem (1.1) dose not admit any global solution provided $I\left(u_{0}\right)<0$.

\section{Critical initial energy $J\left(u_{0}\right)=d$}

In this section, we prove the global existence and finite time blow-up of solutions for problem (1.1) with the critical initial condition $J\left(u_{0}\right)=d$.

Theorem 5.1 (Global existence for $\left.J\left(u_{0}\right)=d\right)$. Let $u_{0} \in \mathcal{H}_{2,0}^{1, \frac{n}{2}}(\mathbb{B})$. Suppose $J\left(u_{0}\right)=d$ and $I\left(u_{0}\right) \geq 0$. Then problem $(1.1)$ has a global weak solution $u \in L^{\infty}\left(0, \infty ; \mathcal{H}_{2,0}^{1, \frac{n}{2}}(\mathbb{B})\right)$ with $u_{t} \in L^{2}\left(0, \infty ; \mathcal{H}_{2,0}^{1, \frac{n}{2}}(\mathbb{B})\right)$, and $u(t) \in \bar{W}=W \cup \partial W$ for $0 \leq t<\infty$. 
Proof. Let $\mu_{m}=1-\frac{1}{m}$ and $u_{0 m}=\mu_{m} u_{0}, m=2,3, \cdots$. We consider the following problem

$$
\begin{cases}u_{t}-\triangle_{\mathbb{B}} u_{t}-\triangle_{\mathbb{B}} u=|u|^{p-1} u, & x \in \operatorname{int} \mathbb{B}, t>0, \\ u(0, x)=u_{0 m}(x), & x \in \operatorname{int} \mathbb{B}, \\ u(t, x)=0, & x \in \partial \mathbb{B}, t \geq 0 .\end{cases}
$$

From $I\left(u_{0}\right) \geq 0$ and Lemma 3.1, we have $\lambda^{*}=\lambda^{*}\left(u_{0}\right) \geq 1$. Thus, we get $I\left(u_{0 m}\right)=I\left(\mu_{m} u_{0}\right)>0$ and $J\left(u_{0 m}\right)=J\left(\mu_{m} u_{0}\right)<J\left(u_{0}\right)<d$. So it follows from Theorem 4.1 that, for each $m$, problem (5.1) admits a global weak solution $u_{m}(t) \in L^{\infty}\left(0, \infty ; \mathcal{H}_{2,0}^{1, \frac{n}{2}}(\mathbb{B})\right)$ with $u_{m t} \in L^{2}\left(0, \infty ; \mathcal{H}_{2,0}^{1, \frac{n}{2}}(\mathbb{B})\right)$ and $u_{m t} \in W$ for $0 \leq t<\infty$, satisfying

$$
\left(u_{m t}, v\right)_{2}+\left(\nabla_{\mathbb{B}} u_{m}, \nabla_{\mathbb{B}} v\right)_{2}+\left(\nabla_{\mathbb{B}} u_{m t}, \nabla_{\mathbb{B}} v\right)_{2}=\left(u_{m}^{p}, v\right)_{2} \text { for any } v \in \mathcal{H}_{2,0}^{1, \frac{n}{2}}(\mathbb{B})
$$

and

$$
\int_{0}^{t}\left\|u_{m \tau}\right\|_{L_{2}^{\frac{n}{2}(\mathbb{B})}}^{2} d \tau+\int_{0}^{t}\left\|\nabla_{\mathbb{B}} u_{m \tau}\right\|_{L_{2}^{\frac{n}{2}}(\mathbb{B})}^{2} d \tau+J\left(u_{m}\right) \leq J\left(u_{0 m}\right)<J\left(u_{0}\right)=d \text { for } t \in(0, \infty) .
$$

By a direct computation we can see that

$$
\int_{0}^{t}\left\|u_{m \tau}\right\|_{L_{2}^{\frac{n}{2}(\mathbb{B})}}^{2} d \tau+\int_{0}^{t}\left\|\nabla_{\mathbb{B}} u_{m \tau}\right\|_{L_{2}^{\frac{n}{2}(\mathbb{B})}}^{2} d \tau+\frac{p-1}{2(p+1)}\left\|\nabla_{\mathbb{B}} u_{m}\right\|_{L_{2}^{\frac{n}{2}(\mathbb{B})}}^{2}+\frac{1}{p+1} I\left(u_{m}\right)<d .
$$

Since $I\left(u_{m}\right) \geq 0$, we can deduce (4.5), (4.6), (4.7) for each $m$. Hence there exists a $u$ and a subsequence still denoted as $\left\{u_{m}\right\}$, such that, as $m \rightarrow \infty$,

$$
\begin{aligned}
& u_{m} \rightarrow u \text { in } L^{\infty}\left(0, \infty ; \mathcal{H}_{2,0}^{1, \frac{n}{2}}(\mathbb{B})\right) \text { weakly star and a.e. in } \operatorname{int} \mathbb{B} \times[0, \infty), \\
& u_{m t} \rightarrow u_{t} \text { in } L^{2}\left(0, \infty ; \mathcal{H}_{2,0}^{1, \frac{n}{2}}(\mathbb{B})\right) \text { weakly star, } \\
& u_{m}^{p} \rightarrow u^{p} \text { in } L^{\infty}\left(0, \infty ; L_{\frac{p+1}{p}}^{\frac{p n}{p+1}}(\mathbb{B})\right) \text { weakly star and a.e. in int } \mathbb{B} \times[0, \infty) .
\end{aligned}
$$

The proof of global existence for the solution is the same as that in the first part of the Theorem 4.1.

Theorem 5.2. Let $u_{0} \in \mathcal{H}_{2,0}^{1, \frac{n}{2}}(\mathbb{B})$. Assume that $J\left(u_{0}\right)=d$ and $I\left(u_{0}\right) \geq 0$. Then, for the global weak solution $u$ of problem (1.1), there exist constants $C>0$ and $t_{1}>0$ such that

$$
\|u\|_{L_{2}^{\frac{n}{2}(\mathbb{B})}}^{2}+\left\|\nabla_{\mathbb{B}} u\right\|_{L_{2}^{\frac{n}{2}(\mathbb{B})}}^{2} \leq\left(\left\|u\left(t_{1}\right)\right\|_{L_{2}^{\frac{n}{2}(\mathbb{B})}}^{2}+\left\|\nabla_{\mathbb{B}} u\left(t_{1}\right)\right\|_{L_{2}^{\frac{n}{2}(\mathbb{B})}}^{2}\right) e^{-C\left(t-t_{1}\right)}, \quad t_{1} \leq t<\infty .
$$

Proof. First, Theorem 4.1 gives the existence of a global weak solution for problem (1.1). In addition, from (3.8), Theorem 4.1, and Theorem 5.1, it follows that if $u(t)$ is a global weak solution of problem (1.1) with $J\left(u_{0}\right)=d, I\left(u_{0}\right) \geq 0$, we must have $I(u) \geq 0$ for $0 \leq t<\infty$. Next, we consider the following two cases.

(i) Assume that $I(u)>0$ for $0 \leq t<\infty$. Then from $\left(u_{t}, u\right)_{2}+\left(\nabla_{\mathbb{B}} u_{t}, \nabla_{\mathbb{B}} u\right)_{2}=-I(u)<0$, it follows that $\left\|u_{t}\right\|_{L_{2}^{\frac{n}{2}}(\mathbb{B})}>0$ and $\left\|\nabla_{\mathbb{B}} u_{t}\right\|_{L_{2}^{\frac{n}{2}}(\mathbb{B})}>0$ and $\int_{0}^{t}\left(\left\|u_{\tau}\right\|_{L_{2}^{\frac{n}{2}}(\mathbb{B})}^{2}+\left\|\nabla_{\mathbb{B}} u_{\tau}\right\|_{L_{2}^{\frac{n}{2}}(\mathbb{B})}^{2}\right) d \tau$ is strictly increasing for $0 \leq t<\infty$. Taking any $t_{1}>0$ and letting

$$
d_{1}=J\left(u\left(t_{1}\right)\right)=J\left(u_{0}\right)-\int_{0}^{t}\left\|u_{\tau}\right\|_{L_{2}^{\frac{n}{2}}(\mathbb{B})}^{2}+\left\|\nabla_{\mathbb{B}} u_{\tau}\right\|_{L_{2}^{\frac{n}{2}(\mathbb{B})}}^{2} d \tau
$$

then by the energy inequality we get $0<J(u) \leq d_{1}<d$ for $t_{1} \leq t<\infty$. Similar to the proof of Theorem 4.1, we can deduce the exponential decay (5.3) if we take $t=t_{1}$ as the initial time. 
(ii) Assume that there exists a $t_{1}>0$ such that $I\left(u\left(t_{1}\right)\right)=0$ and $I(u)>0$ for $0 \leq t<t_{1}$. We also have $\left\|u_{t}\right\|_{L_{2}^{\frac{n}{2}(\mathbb{B})}}>0,\left\|\nabla_{\mathbb{B}} u_{t}\right\|_{L_{2}^{\frac{n}{2}(\mathbb{B})}}>0$ and $\int_{0}^{t}\left(\left\|u_{\tau}\right\|_{L_{2}^{\frac{n}{2}(\mathbb{B})}}^{2}+\left\|\nabla_{\mathbb{B}} u_{\tau}\right\|_{L_{2}^{\frac{n}{2}}(\mathbb{B})}^{2}\right) d \tau$ is strictly increasing for $0 \leq t<t_{1}$. By (3.8), we have

$$
J\left(u\left(t_{1}\right)\right) \leq J\left(u_{0}\right)-\int_{0}^{t}\left\|u_{\tau}\right\|_{L_{2}^{\frac{n}{2}(\mathbb{B})}}^{2}+\left\|\nabla_{\mathbb{B}} u_{\tau}\right\|_{L_{2}^{\frac{n}{2}(\mathbb{B})}}^{2} d \tau<d
$$

and $\left\|\nabla_{\mathbb{B}} u\left(t_{1}\right)\right\|_{L_{2}^{\frac{n}{2}(\mathbb{B})}}^{2}=0$. That means $J\left(u\left(t_{1}\right)\right)=0$, then we get $J(u) \leq 0$ for $t_{1} \leq t<\infty$ from

$$
J(u)+\int_{t_{1}}^{t}\left\|u_{\tau}\right\|_{L_{2}^{\frac{n}{2}(\mathbb{B})}}^{2}+\left\|\nabla_{\mathbb{B}} u_{\tau}\right\|_{L_{2}^{\frac{n}{2}(\mathbb{B})}}^{2} d \tau \leq J\left(u\left(t_{1}\right)\right), \quad t_{1} \leq t<\infty .
$$

Hence from

$$
\begin{aligned}
\frac{1}{2}\left\|\nabla_{\mathbb{B}} u\right\|_{L_{2}^{\frac{n}{2}(\mathbb{B})}}^{2} & \leq \frac{1}{p+1} \int_{\mathbb{B}}|u|^{p+1} \frac{d x_{1}}{x_{1}} d x^{\prime} \\
& \leq \frac{1}{p+1} C_{*}^{p+1}\left\|\nabla_{\mathbb{B}} u\right\|_{L_{2}^{\frac{n}{2}(\mathbb{B})}}^{p-1}\left\|\nabla_{\mathbb{B}} u\right\|_{L_{2}^{\frac{n}{2}(\mathbb{B})}}^{2},
\end{aligned}
$$

it follows that either $\left\|\nabla_{\mathbb{B}} u\right\|_{L_{2}^{\frac{n}{2}(\mathbb{B})}}=0$ for $t_{1} \leq t<\infty$, hence (5.3) holds; or $\left\|\nabla_{\mathbb{B}} u\right\|_{L_{2}^{\frac{n}{2}(\mathbb{B})}}^{2} \geq$ $\left(\frac{p+1}{2 C_{*}^{p+1}}\right)^{\frac{1}{p-1}}$, for $t_{1} \leq t<\infty$, which is impossible since $\left\|\nabla_{\mathbb{B}} u\left(t_{1}\right)\right\|_{L_{2}^{\frac{n}{2}}(\mathbb{B})}=0$. Thereby, we conclude $\|u\|_{L_{2}^{\frac{n}{2}(\mathbb{B})}}^{2}+\left\|\nabla_{\mathbb{B}} u\right\|_{L_{2}^{\frac{n}{2}(\mathbb{B})}}^{2} \equiv 0$ for $t_{1} \leq t<\infty$. This completes the proof.

Theorem 5.3 (Blow-up for $J\left(u_{0}\right)=d$ ). Let $u_{0} \in \mathcal{H}_{2,0}^{1, \frac{n}{2}}(\mathbb{B})$. Suppose $J\left(u_{0}\right)=d$ and $I\left(u_{0}\right)<0$. Then the existence time of the weak solution for problem (1.1) is finite, i.e., the maximal existence time $T_{\max }$ is finite and

$$
\lim _{t \rightarrow T_{\text {max }}^{-}} \int_{0}^{t}\left(\|u\|_{L_{2}^{\frac{n}{2}(\mathbb{B})}}^{2}+\left\|\nabla_{\mathbb{B}} u\right\|_{L_{2}^{\frac{n}{2}(\mathbb{B})}}^{2}\right) d \tau=+\infty .
$$

Proof. Let $u(t)$ be any weak solution of problem $(1.1)$ with $J\left(u_{0}\right)=d, I\left(u_{0}\right)<0$. By contradiction, we suppose that $u(t)$ is global, then $T_{\max }=\infty$. For any $T>0$ and for all $t \in[0, T]$, we define

$$
L(t)=\int_{0}^{t}\|u(\tau)\|_{L_{2}^{\frac{n}{2}(\mathbb{B})}}^{2} d \tau+\int_{0}^{t}\left\|\nabla_{\mathbb{B}} u(\tau)\right\|_{L_{2}^{\frac{n}{2}}(\mathbb{B})}^{2} d \tau+(T-t)\left(\left\|u_{0}\right\|_{L_{2}^{\frac{n}{2}}(\mathbb{B})}^{2}+\left\|\nabla_{\mathbb{B}} u_{0}\right\|_{L_{2}^{\frac{n}{2}}(\mathbb{B})}^{2}\right)+b\left(t+T_{0}\right)^{2}
$$

where $b$ and $T_{0}$ are positive constants which will be specified later. Furthermore,

$$
\begin{aligned}
L^{\prime}(t) & =\|u(t)\|_{L_{2}^{\frac{n}{2}(\mathbb{B})}}^{2}+\left\|\nabla_{\mathbb{B}} u(t)\right\|_{L_{2}^{\frac{n}{2}(\mathbb{B})}}^{2}-\left(\left\|u_{0}\right\|_{L_{2}^{\frac{n}{2}(\mathbb{B})}}^{2}+\left\|\nabla_{\mathbb{B}} u_{0}\right\|_{L_{2}^{\frac{n}{2}(\mathbb{B})}}^{2}\right)+2 b\left(t+T_{0}\right) \\
& =2 \int_{0}^{t}\left(u(\tau), u_{\tau}(\tau)\right)_{2} d \tau+2 \int_{0}^{t}\left(\nabla_{\mathbb{B}} u(\tau), \nabla_{\mathbb{B}} u_{\tau}(\tau)\right)_{2} d \tau+2 b\left(t+T_{0}\right)
\end{aligned}
$$

and, consequently,

$$
L^{\prime \prime}(t)=2 \int_{\mathbb{B}} u(t) u_{t}(t) \frac{d x_{1}}{x_{1}} d x^{\prime}+2 \int_{\mathbb{B}} \nabla_{\mathbb{B}} u(t) \nabla_{\mathbb{B}} u_{t}(t) \frac{d x_{1}}{x_{1}} d x^{\prime}+2 b,
$$

for almost every $t \in[0, T]$. Therefore, we get

$$
L(t) L^{\prime \prime}(t)-\frac{p+1}{2} L^{\prime}(t)^{2}
$$




$$
\begin{aligned}
= & L(t) L^{\prime \prime}(t)+2(p+1)\left[\eta(t)-\left(L(t)-(T-t)\left(\left\|u_{0}\right\|_{L_{2}^{\frac{n}{2}(\mathbb{B})}}^{2}+\left\|\nabla_{\mathbb{B}} u_{0}\right\|_{L_{2}^{\frac{n}{2}(\mathbb{B})}}^{2}\right)\right)\right]\left(\int_{0}^{t}\left\|u_{\tau}(\tau)\right\|_{L_{2}^{\frac{n}{2}(\mathbb{B})}}^{2} d \tau\right. \\
& \left.+\int_{0}^{t}\left\|\nabla_{\mathbb{B}} u_{\tau}(\tau)\right\|_{L_{2}^{\frac{n}{2}}(\mathbb{B})}^{2} d \tau+b\right)
\end{aligned}
$$

where $\eta:[0, T] \rightarrow \mathbb{R}_{+}$is the function defined by

$$
\begin{array}{r}
\eta=\left(\int_{0}^{t}\|u(\tau)\|_{L_{2}^{\frac{n}{2}(\mathbb{B})}}^{2} d \tau+\int_{0}^{t}\left\|\nabla_{\mathbb{B}} u(\tau)\right\|_{L_{2}^{\frac{n}{2}(\mathbb{B})}}^{2} d \tau+b\right)\left(\int_{0}^{t}\left\|u_{\tau}(\tau)\right\|_{L_{2}^{\frac{n}{2}(\mathbb{B})}}^{2} d \tau+\int_{0}^{t}\left\|\nabla_{\mathbb{B}} u_{\tau}(\tau)\right\|_{L_{2}^{\frac{n}{2}(\mathbb{B})}}^{2} d \tau\right. \\
\left.+b\left(t+T_{0}\right)^{2}\right)-\left(\int_{0}^{t}\left(u(\tau), u_{\tau}(\tau)\right)_{2} d \tau+\int_{0}^{t}\left(\nabla_{\mathbb{B}} u(\tau), \nabla_{\mathbb{B}} u_{\tau}(\tau)\right)_{2} d \tau+b\left(t+T_{0}\right)\right)^{2} \geq 0 .
\end{array}
$$

As a consequence, we read the following differential inequality

$$
\begin{aligned}
L(t) L^{\prime \prime}(t)-\frac{p+1}{2} L^{\prime}(t)^{2} & \geq L(t) L^{\prime \prime}(t)-2(p+1) L(t)\left(\int_{0}^{t}\left\|u_{\tau}(\tau)\right\|_{L_{2}^{\frac{n}{2}(\mathbb{B})}}^{2} d \tau+\int_{0}^{t}\left\|\nabla_{\mathbb{B}} u_{\tau}(\tau)\right\|_{L_{2}^{\frac{n}{2}(\mathbb{B})}}^{2} d \tau+b\right) \\
& =L(t) \xi(t),
\end{aligned}
$$

for almost every $t \in[0, T]$, where $\xi:[0, T] \rightarrow \mathbb{R}_{+}$is the map defined by

$$
\begin{aligned}
\xi(t)=2 \int_{\mathbb{B}} u(t) u_{t}(t) \frac{d x_{1}}{x_{1}} d x^{\prime}+2 \int_{\mathbb{B}} \nabla_{\mathbb{B}} u(t) \nabla_{\mathbb{B}} u_{t}(t) \frac{d x_{1}}{x_{1}} d x^{\prime}-2(p+1) \int_{0}^{t}\left\|u_{\tau}(\tau)\right\|_{L_{2}^{\frac{n}{2}(\mathbb{B})}}^{2} d \tau \\
-2(p+1) \int_{0}^{t}\left\|\nabla_{\mathbb{B}} u_{\tau}(\tau)\right\|_{L_{2}^{\frac{n}{2}}(\mathbb{B})}^{2} d \tau-2 p b .
\end{aligned}
$$

By (1.1) and (3.8), we have

$$
\begin{aligned}
\xi(t)= & 2 \int_{\mathbb{B}}\left(u^{p+1}-\left\|\nabla_{\mathbb{B}} u\right\|^{2}\right) \frac{d x_{1}}{x_{1}} d x^{\prime}-2(p+1) \int_{0}^{t}\left\|u_{\tau}(\tau)\right\|_{L_{2}^{\frac{n}{2}}(\mathbb{B})}^{2} d \tau \\
& -2(p+1) \int_{0}^{t}\left\|\nabla_{\mathbb{B}} u_{\tau}(\tau)\right\|_{L_{2}^{\frac{n}{2}}(\mathbb{B})}^{2} d \tau-2 p b \\
\geq & 2 \int_{\mathbb{B}}\left(u^{p+1}-\left\|\nabla_{\mathbb{B}} u\right\|^{2}\right) \frac{d x_{1}}{x_{1}} d x^{\prime}-2(p+1) J\left(u_{0}\right)+2(p+1) J(u(t))-2 p b .
\end{aligned}
$$

From $J\left(u_{0}\right)=d$, it follows

$$
\xi(t) \geq(p-1)\left\|\nabla_{\mathbb{B}} u\right\|_{L_{2}^{\frac{n}{2}}(\mathbb{B})}^{2}-2(p+1) d-2 p b .
$$

The reminder of the proof is the same as those of Theorem 4.2, therefore we omit it.

\section{ACKNOWLEDGMENTS}

This work was supported by the National Natural Science Foundation of China (Grant No. 11301277), the Natural Science Foundation of Jiangsu Province (Grant No. BK20151523), the Six Talent Peaks Project in Jiangsu Province (Grant No. 2015-XCL-020), and the Qing Lan Project of Jiangsu Province.

\section{REFERENCES}

[1] M. Alimohammady and M. K. Kalleji, Existence result for a class of semilinear totally characteristic hypoelliptic equations with conical degeneration, J. Funct. Anal. 265 (2013), no. 10, 2331-2356.

[2] T. B. Benjamin, J. L. Bona and J. J. Mahony, Model equations for long waves in nonlinear dispersive systems, Philos. Trans. Roy. Soc. London Ser. A 272 (1972), no. 1220, 47-78.

[3] M. M. Cavalcanti, V. N. Domingos Cavalcanti and I. Lasiecka, Well-posedness and optimal decay rates for the wave equation with nonlinear boundary damping - source interaction, J. Differential Equations 236 (2007), no. 2, 407-459. 
[4] M. M. Cavalcanti et al., Asymptotic stability of the wave equation on compact manifolds and locally distributed damping: a sharp result, Arch. Ration. Mech. Anal. 197 (2010), no. 3, 925-964.

[5] H. Chen and G. Liu, Global existence and nonexistence for semilinear parabolic equations with conical degeneration, J. Pseudo-Differ. Oper. Appl. 3 (2012), no. 3, 329-349.

[6] H. Chen, X. Liu and Y. Wei, Cone Sobolev inequality and Dirichlet problem for nonlinear elliptic equations on a manifold with conical singularities, Calc. Var. Partial Differential Equations 43 (2012), no. 3-4, 463-484.

[7] H. Chen, X. Liu and Y. Wei, Existence theorem for a class of semilinear totally characteristic elliptic equations with critical cone Sobolev exponents, Ann. Global Anal. Geom. 39 (2011), no. 1, 27-43.

[8] H. Chen and N. Liu, Asymptotic stability and blow-up of solutions for semi-linear edge-degenerate parabolic equations with singular potentials, Discrete Contin. Dyn. Syst. 36 (2016), no. 2, 661-682.

[9] H. Di, Y. Shang and X. Peng, Global existence and nonexistence of solutions for a viscoelastic wave equation with nonlinear boundary source term, Math. Nachr. 289 (2016), no. 11-12, 1408-1432.

[10] H. Fan and X. Liu, Multiple positive solutions for degenerate elliptic equations with critical cone Sobolev exponents on singular manifolds, Electron. J. Differential Equations 2013 (2013), no. 181, 1-22.

[11] M.O. Korpusov and A.G. Sveshnikov, Blow-up of solutions of Sobolev-type nonlinear equations with cubic sources, Differ. Equ. 42 (2006), no. 3, 431-443.

[12] H. A. Levine, Some nonexistence and instability theorems for solutions of formally parabolic equations of the form $P u_{t}=-A u+\mathscr{F}(u)$, Arch. Rational Mech. Anal. 51 (1973), no. 5, 371-386.

[13] W. J. Liu, Global existence, asymptotic behavior and blow-up of solutions for a viscoelastic equation with strong damping and nonlinear source, Topol. Methods Nonlinear Anal. 36 (2010), no. 1, 153-178.

[14] W. J. Liu, Global existence, asymptotic behavior and blow up of solutions for coupled Klein-Gordon equations with damping terms, Nonlinear Anal. 73 (2010), no. 1, 244-255.

[15] W. J. Liu, Y. Sun and G. Li, On decay and blow-up of solutions for a singular nonlocal viscoelastic problem with a nonlinear source term, Topol. Methods Nonlinear Anal. (2017), in press. DOI: 10.12775/TMNA.2016.077

[16] Y. Liu and J. Zhao, On potential wells and applications to semilinear hyperbolic equations and parabolic equations, Nonlinear Anal. 64 (2006), no. 12, 2665-2687.

[17] Y. Liu and R. Xu, Wave equations and reaction-diffusion equations with several nonlinear source terms of different sign, Discrete Contin. Dyn. Syst. Ser. B 7 (2007), no. 1, 155-173.

[18] P. Luo, Blow-up phenomena for a pseudo-parabolic equation, Math. Methods Appl. Sci. 38 (2015), no. 12, $2636-2641$.

[19] V. Padrón, Effect of aggregation on population revovery modeled by a forward-backward pseudoparabolic equation, Trans. Amer. Math. Soc. 356 (2004), no. 7, 2739-2756.

[20] L. E. Payne and D. H. Sattinger, Saddle points and instability of nonlinear hyperbolic equations, Israel J. Math. 22 (1975), no. 3-4, 273-303.

[21] D. H. Sattinger, On global solution of nonlinear hyperbolic equations, Arch. Rational Mech. Anal. 30 (1968), no. 2, $148-172$.

[22] F. Sun, M. Wang and H. Li, Global and blow-up solutions for a quasilinear hyperbolic equation with strong damping, Nonlinear Anal. 73 (2010), no. 5, 1408-1425.

[23] F. Tahamtani and A. Peyravi, Asymptotic behavior and blow-up of solutions for a nonlinear viscoelastic wave equation with boundary dissipation, Taiwanese J. Math. 17 (2013), no. 6, 1921-1943.

[24] R. Xu and J. Su, Global existence and finite time blow-up for a class of semilinear pseudo-parabolic equations, J. Funct. Anal. 264 (2013), no. 12, 2732-2763.

(G. Li) College of Mathematics and Statistics, Nanjing University of Information Science and Technology, NANJing 210044, China

E-mail address: ligang@nuist.edu.cn

(J. Y. Yu) College of Mathematics and Statistics, Nanjing University of Information Science and TechnologY, NANJing 210044, China

E-mail address: yjyamxp@outlook.com

(W. J. Liu) College of Mathematics and Statistics, Nanjing University of Information Science and TechnolOGY, NANJING 210044, CHINA

E-mail address: wjliu@nuist.edu.cn

(C) 2017 by the authors. Licensee Preprints, Basel, Switzerland. This article is an open access article distributed under the terms and conditions of the Creative Commons by Attribution (CC-BY) license (http://creativecommons.org/licenses/by/4.0/). 